Research Article

\title{
Generalised Presic Type Operators in Modular Metric Space and an Application to Integral Equations of Caratheodory Type Functions
}

\author{
Fahad Sameer Alshammari $\mathbb{D}^{1},{ }^{1}$ K. P. Reshma, ${ }^{2}$ Rajagopalan R., ${ }^{1}$ and Reny George $\mathbb{D}^{1}$ \\ ${ }^{1}$ Department of Mathematics, College of Science and Humanities in Alkharj, Prince Sattam bin Abdulaziz University, \\ Al-Kharj 11942, Saudi Arabia \\ ${ }^{2}$ Department of Mathematics, Rungta College of Engineering and Technology, Bhilai, Chhattisgarh, India
}

Correspondence should be addressed to Fahad Sameer Alshammari; f.alshammari@psau.edu.sa and Reny George;

renygeorge02@yahoo.com

Received 7 June 2021; Accepted 7 September 2021; Published 7 October 2021

Academic Editor: Naeem Saleem

Copyright (c) 2021 Fahad Sameer Alshammari et al. This is an open access article distributed under the Creative Commons Attribution License, which permits unrestricted use, distribution, and reproduction in any medium, provided the original work is properly cited.

Extending the Presic type operators to modular spaces, we introduce generalised Presic type $w$-contractive mappings and strongly $w$-contractive mappings in a modular metric space and establish fixed-point theorems for such contractions in modular spaces. Ulam-Hyers stability of the fixed-point equation involving Presic type operators is also discussed. Our results extend and generalise some known results in the literature. The results are supported by appropriate example and an application to Caratheodory type integral equation.

\section{Introduction}

Maurice Ren Fréchet [1] introduced the general and axiomatic form of distance as " $L-$ space." Felix Hausdorff [2] reexamined it as a metric space in the setting of points which has been refined, discussed, and generalised in numerous ways. Bakhtin [3], Branciari [4], George et al. [5], and Mitrović and Radenović [6] introduced the notions of a $b$-metric, a rectangular metric, a rectangular $b$-metric, and $b_{v}^{s}$-metric, respectively. The basic concepts and theory of modular space was formulated in [7]. Later, in [8], the authors established fixed-point theorems in a modular function space. In 2008, Chistyakov [9] introduced the notion of a modular metric and the corresponding modular space. Chistyakov, in [10], established the existence of fixed point for contractive maps and strongly contractive maps in modular metric spaces. Umit et al. [11] introduced Bogin type $w$-contractions and proved fixed-point theorems for such contractions in a $w$-complete modular metric space and provided application to antiperiodic boundary value problems. Furthermore, we see that Chaipunya et al. [12] introduced Geraghty type theorems and Turkoglu and Kilinc [13] introduced Caristi type theorems in a modular metric space and gave its applications in integral equations. For more results of fixed-point theorems in modular metric space and some applications of fixed-point theorems, readers may refer to [14-20]. On the other hand, Presic $[21,22]$ extended the Banach contraction principle to the product of a finite number of metric spaces and later Ciric and Presic [23] generalised the result of Presic [21] and proved the following.

Theorem 1. Let $(X, d)$ be a metric space, $k$ be a positive integer, and $T: X^{k} \longrightarrow X$ be a mapping satisfying the following condition: 


$$
d\left(T\left(x_{1}, x_{2}, \ldots, x_{k}\right), T\left(x_{2}, x_{3}, \ldots, x_{k+1}\right)\right) \leq \lambda \cdot \max \left\{d\left(x_{1}, x_{2}\right), d\left(x_{2}, x_{3}\right), \ldots d\left(x_{k}, x_{k+1}\right)\right.
$$

where $x_{1}, x_{2}, \ldots, x_{k+1}$ are arbitrary elements in $X$ and $\lambda \in(0,1)$. Then, there exists some $x \in X$ such that $x=T(x, x, \ldots, x)$. Moreover, if $x_{1}, x_{2}, \ldots, x_{k}$ are arbitrary points in $X$ and for $n \in N, x_{n+k}=T\left(x_{n}, x_{n+1}, \ldots, x_{n+k-1}\right)$, then the sequence $\left\langle x_{n}\right\rangle$ is convergent and if $\lim _{n \rightarrow \infty} x_{n}=x$, then $x=T(x, x, \ldots, x)$. If, in addition, $T$ satisfies $D(T(u, u, \ldots u), T(v, v, \ldots v))<d(u, v)$, for all $u, v \in X$. Then, $x$ is the unique point satisfying $x=T(x, x, \ldots, x)$.

Later, the above results were further extended and generalised to a $b$-metric space, cone metric space, and cone $b$-metric space by many authors, and fruitful applications were found for such results (see [24-29]). The aim of this work is to introduce generalised Presic type contractions (which includes Ciric-Presic type contraction and Presic type contractions) in modular metric spaces and establish fixed-point theorems for such contraction mappings in a $w$-complete modular metric space. Our results show symmetric transformation of the well-known Ciric-Presic theorem [23] and Presic theorem [21] from ordinary metric space to a modular metric space. We have introduced Ulam-Hyers stability of fixed-point equations involving Presic type operators in a modular metric space. We have also provided an application of our result to prove the existence of solution of an integral equation of Caratheodory type functions. Our results extend and generalise many known results in the literature.

\section{Preliminaries}

The following basic concepts of modular spaces are from $[9,10]$. Let $X \neq \phi$ and $w:(0, \infty) \times X \times X \longrightarrow[0, \infty]$ be a given function. For any $\lambda \in R, x, y \in X$, we write $w(\lambda, x, y)$ as $w_{\lambda}(x, y)$.

Definition 1 (see [10]). We call $w$ a modular metric on $X$ if for all $\lambda, \mu>0$ and $x, y, z \in X$, the following relations hold:

$$
\begin{aligned}
& (\mathrm{mm} 1) w_{\lambda}(x, y)=0 \Leftrightarrow x=y \\
& (\mathrm{~mm} 2) w_{\lambda}(x, y)=w(\lambda, y, x) \\
& (\mathrm{mm} 3) w_{\lambda+\mu}(x, y) \leq w_{\lambda}(x, z)+w_{\mu}(z, y)
\end{aligned}
$$

In this case, $w$ is said to be a metric modular on $X$. Instead of (mm1), if $w$ satisfies only

$$
w_{\lambda}(x, x)=0 \text {, }
$$

for all $\lambda>0$ and $x \in X$, then $w$ is said to be pseudomodular on $X$.

Also, $w$ is strict modular on $X$ if instead of (mm1), it satisfies (2) and for $x, y \in X$, there exists a number $\lambda>0$ (possibly depending on $x$ and $y$ ), such that

$$
w_{\lambda}(x, y)=0 \Longrightarrow x=y \text {. }
$$

Clearly, if $w$ is strict modular, then it is modular, which in turn implies $w$ is pseudomodular on $X$.

A metric modular $w$ on $X$ is convex if it satisfies the (stronger) inequality, in lieu of ( $\mathrm{mm} 3)$ :

$$
w_{\lambda+\mu}(x, y) \leq \frac{\lambda}{\lambda+\mu} w_{\lambda}(x, z)+\frac{\mu}{\lambda+\mu} w_{\mu}(z, y),
$$

for all $\lambda, \mu>0$.

The main property of a modular $w$ on a set $X$ is that (see Section 2.3 of [9]), given $x, y \in X$, the function $w_{\lambda}(x, y)$ is nonincreasing on $(0, \infty)$. If $0<\mu<\lambda$, then $(\mathrm{mm} 1)$ and (mm3) imply

$$
w_{\lambda}(x, y) \leq w_{\lambda-\mu}(x, x)+w_{\mu}(x, y)=w_{\mu}(x, y) .
$$

Definition 2 (see [10]). Given a pseudomodular $w$ on $X$, the two sets

$$
\begin{aligned}
& X_{w}=X_{w}\left(x_{0}\right)=\left\{x \in X: w_{\lambda}\left(x, x_{0}\right) \longrightarrow 0 \text { as } \lambda \longrightarrow \infty\right\}, \\
& X_{w}^{*}=X_{w}^{*}\left(x_{0}\right)=\left\{x \in X: \text { there exists } \lambda=\lambda(x)>0 \text { such that } w_{\lambda}\left(x, x_{0}\right)<\infty\right\},
\end{aligned}
$$

are said to be modular spaces (around $x_{0}$ ).

Clearly, $X_{w} \subset X_{w}^{*}$. Note that if $w$ is metric modular on $X$, then modular space $X_{w}$ can be equipped with a metric generated by $w$ given by

$$
d_{w}(x, y)=\inf \left\{\lambda>0: w_{\lambda}(x, y) \leq \lambda\right\}
$$

for any $x, y \in X_{w}$. Moreover, if $w$ is a convex modular on $X$, then by Section 3.5 and Theorem 3.6 of [9], $X_{w}=X_{w}^{*}$; i.e., the two modular spaces coincide, and this common set can be endowed with a metric $d_{w}^{*}$ :

$$
d_{w}^{*}(x, y)=\inf \left\{\lambda>0: w_{\lambda}(x, y) \leq 1\right\}
$$

Henceforth, $\left(X_{w}, w\right)$ and $\left(X_{w}^{*}, w\right)$ represent modular metric spaces induced by $w$.

Definition 3 (see [10]). Let $w$ be a metric modular on $X$. We have the following:

(i) A sequence $\left\{x_{n}\right\}$ in $X_{w}$ is $w$-convergent (or modular convergent) to some $x \in X$, if there exists $\lambda>0$, such that $\lim _{n \longrightarrow \infty} w_{\lambda}\left(x_{n}, x\right)=0$ 
(ii) A sequence $\left\{x_{n}\right\}$ in $X$ is $w$-Cauchy if $\exists \lambda>0$, possibly dependent on $\left\{x_{n}\right\}$, such that $w_{\lambda}\left(x_{m}, x_{n}\right)=0$ as $m, n \longrightarrow \infty$

(iii) $X$ is $w$-complete if every $w$-Cauchy sequence is $w$-convergent

From [10], we have that $X_{w}$ and $X_{w}^{*}$ are closed with respect to $w$ convergence, if $w$ is pseudomodular on $X$. Also, if $w$ is strict, then the modular limit, if exists, is unique. If $w$ is (not necessarily convex) modular on $X$, then $v_{\lambda}(x, y)=$ $\left(w_{\lambda}(x, y) / \lambda\right)$ is always convex modular on $X$.

\section{Main Results}

In this section, we introduce Presic type operators in modular metric space and prove existence of unique fixed points for such operators.
Let $R_{+}$be the set of real numbers, and consider a function $\Phi: R_{+}^{k} \longrightarrow R_{+}$such that

(a) $\Phi$ is increasing in all variables

(b) $\Phi(u, u, u, \ldots, u) \leq u$, for all $u \in R$

Definition 4. Let $w$ be metric modular on $X$ and $T: X_{w}^{k^{*}} \longrightarrow X_{w}^{*}$ (where $X_{w}^{k^{*}}$ denotes $X_{w}^{*} \times X_{w}^{*} \times \cdots k$ times) for some positive integer $k$. Then, we have the following:

(i) $T$ is a generalised Presic type modular contraction (or a generalised Presic w-contraction), if

$$
w_{\xi \lambda}\left(T\left(x_{1}, x_{2}, \ldots, x_{k}\right), T\left(x_{2}, x_{3}, \ldots, x_{k+1}\right)\right) \leq \Phi\left(w_{\lambda}\left(x_{1}, x_{2}\right), w_{\lambda}\left(x_{2}, x_{3}\right), \ldots, w_{\lambda}\left(x_{k}, x_{k+1}\right)\right)
$$

for some $0<\xi<1$ and $\lambda_{0}>0$ possibly depending on $\xi$ and for all $0<\lambda \leq \lambda_{0}$ and $x_{1}, x_{2}, \ldots, x_{k+1} \in X_{w}^{*}$. (ii) $T$ is a generalised Presic type strongly modular contraction (or a generalised Presic strong w-contraction), if

$$
w_{\xi \lambda}\left(T\left(x_{1}, x_{2}, \ldots, x_{k}\right), T\left(x_{2}, x_{3}, \ldots, x_{k+1}\right)\right) \leq \xi \cdot \Phi\left(w_{\lambda}\left(x_{1}, x_{2}\right), w_{\lambda}\left(x_{2}, x_{3}\right), \ldots, w_{\lambda}\left(x_{k}, x_{k+1}\right)\right)
$$

for some $0<\xi<1$ and $\lambda_{0}>0$ possibly depending on $\xi$ and for all $0<\lambda \leq \lambda_{0}$ and $x_{1}, x_{2}, \ldots, x_{k+1} \in X_{w}^{*}$. (iii) $T$ is a Ciric-Presic type modular contraction (or a Ciric-Presic w-contraction), if

$$
w_{\xi \lambda}\left(T\left(x_{1}, x_{2}, \ldots, x_{k}\right), T\left(x_{2}, x_{3}, \ldots, x_{k+1}\right)\right) \leq \max \left\{w_{\lambda}\left(x_{1}, x_{2}\right), w_{\lambda}\left(x_{2}, x_{3}\right), \ldots, w_{\lambda}\left(x_{k}, x_{k+1}\right)\right\}
$$

for some $0<\xi<1$ and $\lambda_{0}>0$ possibly depending on $\xi$ and for all $0<\lambda \leq \lambda_{0}$ and $x_{1}, x_{2}, \ldots, x_{k+1} \in X_{w}^{*}$. (iv) $T$ is a Ciric-Presic type strongly modular contraction (or a Ciric-Presic strong w-contraction), if

$$
w_{\xi \lambda}\left(T\left(x_{1}, x_{2}, \ldots, x_{k}\right), T\left(x_{2}, x_{3}, \ldots, x_{k+1}\right)\right) \leq \xi \cdot \max \left\{w_{\lambda}\left(x_{1}, x_{2}\right), w_{\lambda}\left(x_{2}, x_{3}\right), \ldots, w_{\lambda}\left(x_{k}, x_{k+1}\right)\right\}
$$

for some $0<\xi<1$ and $\lambda_{0}>0$ possibly depending on $\xi$ and for all $0<\lambda \leq \lambda_{0}$ and $x_{1}, x_{2}, \ldots, x_{k+1} \in X_{w}^{*}$. (v) $T$ is a Presic type modular contraction (or a Presic w-contraction), if there exists $\beta_{i} \geq 0, i=1,2, \ldots, k$, $\sum_{i=1}^{k} \beta_{i}<1$, such that

$$
w_{\xi \lambda}\left(T\left(x_{1}, x_{2}, \ldots, x_{k}\right), T\left(x_{2}, x_{3}, \ldots, x_{k+1}\right)\right) \leq \beta_{1} w_{\lambda}\left(x_{1}, x_{2}\right)+\beta_{2} w_{\lambda}\left(x_{2}, x_{3}\right)+\cdots+\beta_{k} w_{\lambda}\left(x_{k}, x_{k+1}\right),
$$

for some $0<\xi<1$ and $\lambda_{0}>0$ possibly depending on $\xi$ and for all $0<\lambda \leq \lambda_{0}$ and $x_{1}, x_{2}, \ldots, x_{k+1} \in X_{w}^{*}$.
Theorem 2. Let $w$ be a strict metric modular on $X$ and $\left(X_{w}^{*}, w\right)$ be $w$-complete. For any positive integer $k$, let 
$T: X_{w}^{k *} \longrightarrow X_{w}^{*}$ be a generalised Presic type strongly $w$-contractive mapping. If there exists $x_{1}, x_{2}, \ldots, x_{k}$ in $X$, such that $w_{\lambda}\left(x_{1}, x_{2}\right)<\infty, w_{\lambda}\left(x_{2}, x_{3}\right)<\infty, \ldots, w_{\lambda}\left(x_{k-1}, x_{k}\right)$ $<\infty, w_{\lambda}\left(x_{k}, T\left(x_{1}, x_{2}, \ldots, x_{k}\right)\right)<\infty$, then $T$ has a fixed point; that is, there exists an $x \in X$ such that $x=T(x, x$, $\ldots, x)$. Moreover, for any $x_{1}, x_{2}, \ldots, x_{k}$ in $X$, with $w_{\lambda}\left(x_{1}, x_{2}\right)<\infty, w_{\lambda}\left(x_{2}, x_{3}\right)<\infty, \ldots, w_{\lambda}\left(x_{k-1}, x_{k}\right)<\infty, w_{\lambda}$ $\left(x_{k}, T\left(x_{1}, x_{2}, \ldots, x_{k}\right)\right)<\infty$, the sequence $\left\langle x_{n}\right\rangle$ given by $x_{k+n}=T\left(x_{1}, x_{2}, \ldots, x_{k}\right)$ converges to a fixed point of $T$. Furthermore, if $T$ satisfies

$$
w_{\xi \lambda}(T(u, u, \ldots, u), T(v, v, \ldots, v))<w_{\lambda}(u, v),
$$

for all $u, v \in X$ or if $\xi \in(0,(1 / k))$, then the fixed point $x$ is unique.

Proof. For arbitrary elements $x_{1}, x_{2}, \ldots, x_{k}$ in $X$ with $w_{\lambda}\left(x_{1}, x_{2}\right)<\infty, w_{\lambda}\left(x_{2}, x_{3}\right)<\infty, \ldots, w_{\lambda}\left(x_{k-1}, x_{k}\right)<\infty, w_{\lambda}$ $\left(x_{k}, T\left(x_{1}, x_{2}, \ldots, x_{k}\right)\right)<\infty$, define the sequence $\left\langle x_{n}\right\rangle$ in $X$ given by $x_{n+k}=T\left(x_{n}, x_{n+1}, \ldots x_{n+k-1}\right), n \in N$. By the $w$-contractivity of $T$, there exist two numbers $0<\xi<1$ and $\lambda_{0}=\lambda_{0}(\xi)$ such that condition (10) holds. Let $\alpha_{n_{\xi p \lambda}}=w_{\xi^{p} \lambda}\left(x_{n}, x_{n+1}\right)$, where $\lambda \leq \lambda_{0}$. Then, $\xi^{p} \lambda<\lambda<\lambda_{0}$ for any positive integer $p$. Using the method of induction, we will prove that for any nonnegative integer $p$,

$$
\alpha_{n_{\xi p \lambda}} \leq R_{\xi \lambda} \theta^{n},
$$

where $\theta=\xi^{(1 / k)}$ and $R_{\xi \lambda}=\max \left(\left(\alpha_{1_{\lambda}} / \theta\right),\left(\alpha_{2_{\lambda}} / \theta^{2}\right), \ldots\right.$, $\left.\left(\alpha_{k_{\lambda}} / \theta^{k}\right)\right)$. Clearly, by the definition of $R_{\xi \lambda},(15)$ is true for $n=1,2, \ldots, k$ and $p=0$. Let the $k+1$ inequalities $\alpha_{n_{\xi p \lambda}} \leq R_{\xi \lambda} \theta^{n}, \alpha_{(n+1)_{\xi p \lambda}} \leq R_{\xi \lambda} \theta^{n+1}, \ldots, \alpha_{(n+k)_{\xi p \lambda}} \leq R_{\xi \lambda} \theta^{n+k}$ hold true. Then,

$$
\begin{aligned}
& \alpha_{(n+k)_{\xi p+1} \lambda}=w_{\xi^{p+1} \lambda}\left(x_{n+k}, x_{n+k+1}\right) \\
& =w_{\xi^{p+1} \lambda}\left(T\left(x_{n}, x_{n+1}, \ldots, x_{n+k-1}\right), T\left(x_{n+1}, x_{n+2}, \ldots, x_{n+k}\right)\right) \\
& \leq \xi \Phi\left(w_{\xi^{p} \lambda}\left(x_{n}, x_{n+1}\right), w_{\xi^{p} \lambda}\left(x_{n+1}, x_{n+2}\right), \ldots, w_{\xi^{p} \lambda}\left(x_{n+k-1}, x_{n+k}\right)\right)
\end{aligned}
$$

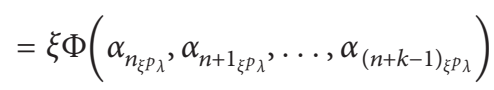

$$
\begin{aligned}
& \leq \xi \Phi\left(R_{\xi \lambda} \theta^{n}, R_{\xi \lambda} \theta^{n+1}, \ldots, R_{\xi \lambda} \theta^{n+k-1}\right) \\
& \leq \xi R_{\xi \lambda} \theta^{n} \\
& =R_{\xi \lambda} \theta^{n+k} \\
& \alpha_{(n+k+1)_{\xi p_{\lambda}}}=w_{\xi^{p} \lambda}\left(x_{n+k+1}, x_{n+k+2}\right) \\
& =w_{\xi^{p} \lambda}\left(T\left(x_{n+1}, x_{n+2}, \ldots, x_{n+k}\right), T\left(x_{n+2}, x_{n+3}, \ldots, x_{n+k+1}\right)\right) \\
& \leq \xi \Phi\left(w_{\xi^{p-1} \lambda}\left(x_{n+1}, x_{n+2}\right), w_{\xi^{p-1} \lambda}\left(x_{n+2}, x_{n+3}\right), \ldots, w_{\xi^{p-1} \lambda}\left(x_{n+k}, x_{n+k+1}\right)\right) \\
& =\xi \Phi\left(\alpha_{n+1_{\xi p-1} \lambda}, \alpha_{n+2_{\xi p-1} \lambda}, \ldots, \alpha_{(n+k)_{\xi p-1} \lambda}\right) \\
& =\xi \Phi\left(\alpha_{n+1_{\xi p \lambda}}, \alpha_{n+2_{\xi P \lambda}}, \ldots \alpha_{(n+k)_{\xi p_{\lambda}}}\right) \\
& \leq \xi \Phi\left(R_{\xi \lambda} \theta^{n+1}, R_{\xi \lambda} \theta^{n+2}, \ldots, R_{\xi \lambda} \theta^{n+k}\right) \\
& \leq \xi R_{\xi \lambda} \theta^{n+1} \\
& =R_{\xi \lambda} \theta^{n+k+1} \text {. }
\end{aligned}
$$

Thus, inductive proof of (15) is complete. Set $\lambda_{1}=(1-\xi) \lambda_{0}$; then, $\xi^{i} \lambda_{1}<\lambda_{1}<\lambda_{0}$. Let integers $n$ and $m$ be such that $n>m$. We set $\lambda=\lambda(n, m)=\xi^{m} \lambda_{1}+\xi^{m+1} \lambda_{1}+\cdots+$ $\xi^{n-1} \lambda_{1}=\xi^{m}\left(\left(1-\xi^{n-m}\right) /(1-\xi)\right) \lambda_{1}$. Then, we have 


$$
\begin{aligned}
w_{\lambda}\left(x_{m}, x_{n}\right) & \leq w_{\xi^{m} \lambda_{1}}\left(x_{m}, x_{m+1}\right)+w_{\xi^{m+1} \lambda_{1}}\left(x_{m+1}, x_{m+2}\right)+\cdots+w_{\xi^{n-1} \lambda_{1}}\left(x_{n-1}, x_{n}\right) \\
& \leq\left[R_{\xi \lambda_{1}} \theta^{m}+R_{\xi \lambda_{1}} \theta^{m+1}+\cdots+R_{\xi \lambda_{1}} \theta^{n}\right] \\
& \leq R_{\xi \lambda_{1}}(\theta)^{m}\left(1+\theta+\theta^{2}+\cdots\right) \\
& =\frac{R_{\xi \lambda_{1}} \theta^{m}}{1-\theta} .
\end{aligned}
$$

Taking into account that $\lambda_{0}=\left(\lambda_{1} /(1-\xi)\right)>\xi^{m}((1-$ $\left.\left.\xi^{n-m}\right) / 1-\xi\right) \lambda_{1}=\lambda$ for all $n>m$, we get

$$
\begin{aligned}
w_{\lambda_{0}}\left(x_{m}, x_{n}\right) & \leq w_{\lambda}\left(x_{m}, x_{n}\right) \\
& \leq \frac{R_{\lambda_{0}}(\xi \theta)^{m}}{1-\theta} \\
& \longrightarrow 0, \quad \text { as } m \longrightarrow \infty .
\end{aligned}
$$

Therefore, $\left\{x_{n}\right\}$ is $w$-Cauchy in $X_{w}^{*}$. By $w$-completeness of $X_{w}^{*}$, there exists $x$ in $X_{w}^{*}$ such that $\lim _{n \longrightarrow \infty} w_{\lambda_{0}}\left(x_{n}, x\right)=0$, and by strictness of $w$, the limit is unique. Let $\epsilon>0$ be arbitrary. Then, we can find a natural number $N$ such that $w_{\lambda_{0}}\left(x_{n}, x_{n+1}\right)<\epsilon$ and $w_{\lambda_{0}}\left(x_{n}, x\right)<\epsilon$ for all $n \geq N$. Then, for any integer $n \geq N$, we have

$$
\begin{aligned}
w_{(1+k \xi) \lambda_{0}}(x, T(x, x, \ldots, x)) \leq & w_{\lambda_{0}}\left(x, x_{n+k}\right)+w_{k \xi \lambda_{0}}\left(x_{n+k}, T(x, x, \ldots, x)\right. \\
= & w_{\lambda_{0}}\left(x, x_{n+k}\right)+w_{\xi \lambda_{0}+\xi \lambda_{0}+\ldots k \text { times }}\left(T\left(x_{n}, x_{n+1}, \ldots, x_{n+k-1}\right), T(x, x, \ldots, x)\right) \\
\leq & w_{\lambda_{0}}\left(x, x_{n+k}\right)+w_{\xi \lambda_{0}}\left(T(x, x, \ldots, x), T\left(x, x, \ldots, x_{n}\right)\right. \\
& +w_{\xi \lambda_{0}}\left(T\left(x, x, \ldots x, x_{n}\right), T\left(x, x, \ldots, x_{n}, x_{n+1}\right)\right. \\
& +\cdots+w_{\xi \lambda_{0}}\left(T\left(x, x_{n}, \ldots, x_{n+k-2}\right), T\left(x_{n}, x_{n+1}, \ldots, x_{n+k-1}\right)\right. \\
\leq & w_{\lambda_{0}}\left(x, x_{n+k}\right)+\xi \Phi\left(w_{\lambda_{0}}(x, x), w_{\lambda_{0}}\left(x, x_{n}\right)\right) \\
& +\xi \Phi\left(w_{\lambda_{0}}(x, x), w_{\lambda_{0}}\left(x, x_{n}\right), w_{\lambda_{0}}\left(x_{n}, x_{n+1}\right)\right)+\ldots \\
& +\xi \Phi\left(w_{\lambda_{0}}\left(x, x_{n}\right), w_{\lambda_{0}}\left(x_{n}, x_{n+1}\right), \ldots, w_{\lambda_{0}}\left(x_{n+k-2}, x_{n+k-1}\right)\right) \\
< & \epsilon .
\end{aligned}
$$

As $\epsilon \longrightarrow 0$, we get $w_{(1+k \xi) \lambda_{0}}(x, T(x, x, \ldots, x))=0$. By the strictness of $w, T(x, x, \ldots, x)=x$. For uniqueness, suppose there exists $y \in X_{w}^{*}$, where $y \neq x$, such that $T(y, \ldots, y)=y$. Then, using (14), we get

$$
w_{\xi \lambda_{0}}(x, y)=w_{\xi \lambda_{0}}(T(x, \ldots, x), T(y, \ldots, y))<w_{\lambda_{0}}(x, y),
$$

a contradiction (as $\xi \lambda_{0}<\lambda_{0}$ ). Thus, the uniqueness of $x$ is established. 
Now, if $\xi \in(0,(1 / k))$, then

$$
\begin{aligned}
w_{\xi \lambda_{0}}(x, y)= & w_{\xi \lambda_{0}}(T(x, \ldots, x), T(y, \ldots, y)) \\
\leq & \frac{\xi \lambda_{0}}{k \xi \lambda_{0}} w_{\left(\xi \lambda_{0} / k\right)}(T(x, x, \ldots, x), T(x, x, \ldots, y) \\
& +\frac{\xi \lambda_{0}}{k \xi \lambda_{0}} w_{\left(\xi \lambda_{0} / k\right)}(T(x, x, \ldots, x, y), T(x, x, \ldots, x, y, y) \\
& +\cdots+\frac{\xi \lambda_{0}}{k \xi \lambda_{0}} w_{\left(\xi \lambda_{0} / k\right)}(T(x, y, \ldots, y), T(y, y, \ldots, y) \\
\leq & \xi \frac{1}{k} \Phi\left(w_{\left(\lambda_{0} / k\right)}(x, x), w_{\left(\lambda_{0} / k\right)}(x, y)\right)+\xi \frac{1}{k} \Phi\left(w_{\left(\lambda_{0} / k\right)}(x, x), w_{\left(\lambda_{0} / k\right)}(x, y), w_{\left(\lambda_{0} / k\right)}(y, y)\right) \\
& \left.+\cdots+\xi_{k}^{1} \Phi_{\left(\lambda_{0} / k\right)}(x, y), w_{\left(\lambda_{0} / k\right)}(y, y)\right) \\
\leq & \xi \frac{k}{k} w_{\left(\lambda_{0} / k\right)}(x, y) \\
< & w\left(\lambda_{0} / k\right)(x, y),
\end{aligned}
$$

a contradiction as $\xi \lambda_{0}<\left(\lambda_{0} / k\right)$. Hence, $T(x, x, \ldots, x)=x$ is unique in $X$.

In the next result, using a convex modular metric, we prove the existence of a fixed point for a generalised Presic type modular contractive mapping.

Theorem 3. Let $w$ be a strict convex modular on $X$ and $\left(X_{w}^{*}, w\right)$ be $w$-complete. For any positive integer $k$, let $T: X_{w}^{k *} \longrightarrow X_{w}^{*}$ be a generalised Presic $w$-contractive mapping. If there exists $x_{1}, x_{2}, \ldots, x_{k}$ in $X$, such that $w_{\lambda}$ $\left(x_{1}, x_{2}\right)<\infty, w_{\lambda}\left(x_{2}, x_{3}\right)<\infty, \ldots, w_{\lambda}\left(x_{k-1}, x_{k}\right)<\infty, w_{\lambda}\left(x_{k}\right.$, $\left.T\left(x_{1}, x_{2}, \ldots, x_{k}\right)\right)<\infty$, then $T$ has a fixed point; that is, there exists an $x \in X$ such that $x=T(x, x, \ldots, x)$. Moreover, for any $x_{1}, x_{2}, \ldots, x_{k}$ in $X$, with $w_{\lambda}\left(x_{1}, x_{2}\right)<\infty, w_{\lambda}\left(x_{2}\right.$, $\left.x_{3}\right)<\infty, \ldots, w_{\lambda}\left(x_{k-1}, x_{k}\right)<\infty, w_{\lambda}\left(x_{k}, T\left(x_{1}, x_{2}, \ldots, x_{k}\right)\right)<$ $\infty$, the sequence $\left\langle x_{n}\right\rangle$ given by $x_{k+n}=T\left(x_{1}, x_{2}, \ldots, x_{k}\right)$ converges to a fixed point of $T$. Furthermore, if $T$ satisfies

$$
w_{\xi \lambda}(T(u, u, \ldots, u), T(v, v, \ldots, v))<w_{\lambda}(u, v),
$$

for all $u, v \in X$ or if $\xi \in(0,(1 / k))$, then the fixed point $x$ is unique.

Proof. We take $u_{\lambda}(x, y)=\lambda . w_{\lambda}(x, y)$ for all $x, y \in X$ and $\lambda>0$. Then, for all $\lambda, \mu>0$ and $x, y, z \in X$, we have

$$
\begin{aligned}
u_{\lambda+\mu}(x, y) & =(\lambda+\mu) w_{\lambda+\mu}(x, y) \\
& \leq(\lambda+\mu)\left\{\frac { \lambda } { \lambda + \mu } \left(w_{\lambda}(x, z)+\frac{\mu}{\lambda+\mu}\left(w_{\mu}(y, z)\right.\right.\right. \\
& =\lambda \cdot w_{\lambda}(x, z)+\mu \cdot w_{\mu}(y, z) \\
& =u_{\lambda}(x, z)+u_{\mu}(y, z) .
\end{aligned}
$$

So, $u$ is strict modular on $X$. Also, by (9), for all $0<\lambda \leq \lambda_{0}$ and $x_{1}, x_{2}, \ldots, x_{k+1} \in X_{w}^{*}$, we have 


$$
\begin{aligned}
u_{\xi \lambda}\left(T\left(x_{1}, x_{2}, \ldots, x_{k}\right), T\left(x_{2}, x_{3}, \ldots, x_{k+1}\right)\right) & =\xi \lambda w_{\xi \lambda}\left(T\left(x_{1}, x_{2}, \ldots, x_{k}\right), T\left(x_{2}, x_{3}, \ldots, x_{k+1}\right)\right) \\
& \leq \xi \lambda \Phi\left(w_{\lambda}\left(x_{1}, x_{2}\right), w_{\lambda}\left(x_{2}, x_{3}\right), \ldots, w_{\lambda}\left(x_{k}, x_{k+1}\right)\right) \\
& =\xi \Phi\left(\lambda w_{\lambda}\left(x_{1}, x_{2}\right), \lambda w_{\lambda}\left(x_{2}, x_{3}\right), \ldots, \lambda w_{\lambda}\left(x_{k}, x_{k+1}\right)\right) \\
& =\xi \Phi\left(u_{\lambda}\left(x_{1}, x_{2}\right), u_{\lambda}\left(x_{2}, x_{3}\right), \ldots, u_{\lambda}\left(x_{k}, x_{k+1}\right)\right) .
\end{aligned}
$$

Thus, $T$ is a generalised Presic strong $\mathrm{w}$-contraction mapping for the modular $u$. Clearly, $w_{\xi^{p} \lambda}\left(x_{1}, x_{2}\right)<\infty, w_{\xi^{p} \lambda}$ $\left(x_{2}, x_{3}\right)<\infty, \ldots, w_{\xi^{p} \lambda}\left(x_{k-1}, x_{k}\right)<\infty, w_{\xi^{p} \lambda}\left(x_{k}, T\left(x_{1}, x_{2}, \ldots\right.\right.$, $\left.\left.x_{k}\right)\right)<\infty \quad$ implies $u_{\xi^{p} \lambda}\left(x_{1}, x_{2}\right)<\infty, u_{\xi^{p} \lambda}\left(x_{2}, \quad x_{3}\right)<\infty$, $\ldots, u_{\xi^{p} \lambda}\left(x_{k-1}, x_{k}\right)<\infty, u_{\xi^{p} \lambda}\left(x_{k}, T\left(x_{1}, x_{2}, \ldots, x_{k}\right)\right)<\infty$. Вy Theorem 2, there exists $x \in X$ such that $u_{(1+k \xi) \lambda_{0}}$ $(x, T(x, x, \ldots, x))=0$. The remaining part of the proof follows the same line as in Theorem 2.

Taking $\Phi\left(u_{1}, u_{2}, \ldots, u_{k}\right)=\max \left\{u_{1}, u_{2}, \ldots, u_{k}\right\} \quad$ in Theorems 1 and 2 , we get the following.

Theorem 4. Let $w$ be strict metric modular on $X$ and $\left(X_{w}^{*}, w\right)$ be $w$-complete. For any positive integer $k$, let $T: X_{w}^{k *} \longrightarrow X_{w}^{*}$ be a Ciric-Presic strongly $w$-contractive mapping. If there exists $x_{1}, x_{2}, \ldots, x_{k}$ in $X$, such that $w_{\lambda}\left(x_{1}, x_{2}\right)<\infty, w_{\lambda}\left(x_{2}, x_{3}\right)<\infty, \ldots, w_{\lambda}\left(x_{k-1}, x_{k}\right)<\infty, w_{\lambda}$ $\left(x_{k}, T\left(x_{1}, x_{2}, \ldots, x_{k}\right)\right)<\infty$, then $T$ has a fixed point; that is, there exists an $x \in X$ such that $x=T(x, x, \ldots, x)$. Moreover, for any $x_{1}, x_{2}, \ldots, x_{k}$ in $X$, with $w_{\lambda}\left(x_{1}, x_{2}\right)<\infty$, $w_{\lambda}\left(x_{2}, x_{3}\right)<\infty, \ldots, w_{\lambda}\left(x_{k-1}, x_{k}\right)<\infty, w_{\lambda}\left(x_{k}, \quad T\left(x_{1}, x_{2}\right.\right.$, $\left.\left.\ldots, x_{k}\right)\right)<\infty$, the sequence $\left\langle x_{n}\right\rangle$ given by $x_{k+n}=T$ $\left(x_{1}, x_{2}, \ldots, x_{k}\right)$ converges to a fixed point of T. Furthermore, if $T$ satisfies

$$
w_{\xi \lambda}(T(u, u, \ldots, u), T(v, v, \ldots, v))<w_{\lambda}(u, v),
$$

for all $u, v \in X$ or if $\xi \in(0,(1 / k))$, then the fixed point $x$ is unique.

Theorem 5. Let $w$ be strict convex modular on $X$ and $\left(X_{w}^{*}, w\right)$ be $w$-complete. For any positive integer $k$, let $T: X_{w}^{k *} \longrightarrow X_{w}^{*}$ be a Ciric-Presic $w$-contractive mapping. If there exists $x_{1}, x_{2}, \ldots, x_{k}$ in $X$, such that $w_{\lambda}\left(x_{1}, x_{2}\right)$ $<\infty, w_{\lambda}\left(x_{2}, x_{3}\right)<\infty, \ldots, w_{\lambda}\left(x_{k-1}, x_{k}\right)<\infty, w_{\lambda}\left(x_{k}, T\left(x_{1}\right.\right.$, $\left.\left.x_{2}, \ldots, x_{k}\right)\right)<\infty$, then $T$ has a fixed point; that is, there exists an $x \in X$ such that $x=T(x, x, \ldots, x)$. Moreover, for any $x_{1}, x_{2}, \ldots, x_{k}$ in $X$, with $w_{\lambda}\left(x_{1}, x_{2}\right)<\infty, w_{\lambda}\left(x_{2}, x_{3}\right)$ $<\infty, \ldots, w_{\lambda}\left(x_{k-1}, x_{k}\right)<\infty, w_{\lambda}\left(x_{k}, T\left(x_{1}, x_{2}, \ldots, x_{k}\right)\right)<\infty$, the sequence $\left\langle x_{n}\right\rangle$ given by $x_{k+n}=T\left(x_{1}, x_{2}, \ldots, x_{k}\right)$ converges to a fixed point of $T$. Furthermore, if $T$ satisfies

$$
w_{\xi \lambda}(T(u, u, \ldots, u), T(v, v, \ldots, v))<w_{\lambda}(u, v),
$$

for all $u, v \in X$ or if $\xi \in(0,(1 / k))$, then the fixed point $x$ is unique.

Taking $\Phi\left(u_{1}, u_{2}, \ldots, u_{k}\right)=\beta_{1} u_{1}+\beta_{2} u_{2}, \ldots, \beta_{k} u_{k}$, with $0 \leq \beta_{i}, \sum_{i=1}^{k} \beta_{i}<1$ in Theorem 3, we get the following.

Theorem 6. Let $w$ be strict convex modular on $X$ and $\left(X_{w}^{*}, w\right)$ be $w$-complete. For any positive integer $k$, let $T: X_{w}^{k *} \longrightarrow X_{w}^{*}$ be a Presic type $w$-contractive mapping. If there exists $x_{1}, x_{2}, \ldots, x_{k}$ in $X$, with $w_{\lambda}\left(x_{1}, x_{2}\right)<\infty, w_{\lambda}$ $\left(x_{2}, x_{3}\right)<\infty, \ldots, w_{\lambda}\left(x_{k-1}, x_{k}\right)<\infty, w_{\lambda}\left(x_{k}, T\left(x_{1}, x_{2}, \ldots\right.\right.$, $\left.\left.x_{k}\right)\right)<\infty$, then $T$ has a fixed point; that is, there exists an $x \in X$ such that $x=T(x, x, \ldots, x)$. Moreover, for any $x_{1}, x_{2}, \ldots, x_{k}$ in $X$, with $w_{\lambda}\left(x_{1}, x_{2}\right)<\infty, w_{\lambda}\left(x_{2}, x_{3}\right)$ $<\infty, \ldots, w_{\lambda}\left(x_{k-1}, x_{k}\right)<\infty, w_{\lambda}\left(x_{k}, T\left(x_{1}, x_{2}, \ldots, x_{k}\right)\right)<\infty$, the sequence $\left\langle x_{n}\right\rangle$ given by $x_{k+n}=T\left(x_{1}, x_{2}, \ldots, x_{k}\right)$ converges to a fixed point of $T$. Furthermore, if $T$ satisfies

$$
w_{\xi \lambda}(T(u, u, \ldots, u), T(v, v, \ldots, v))<w_{\lambda}(u, v)
$$

for all $u, v \in X$ or if $\xi \in(0,(1 / k))$, then the fixed point $x$ is unique.

Remark 1 . For $k=1$, Theorems 4 and 5 reduce to Theorem 10 and Theorem 5.4 of [10].

Remark 2. Theorems 4 and 5 extend the results of Ciric and Presic [23] to a modular space.

Remark 3. Theorem 6 extends the result of Presic [21] to a modular space.

Example 1. Let $X=[0,1]$. Define the mapping $w$ : $(0, \infty) \times$ $X \times X \longrightarrow[0, \infty]$ by $w_{\lambda}\left(a_{1}, a_{2}\right)=\left(4 / 15 \lambda^{2}\right)\left|a_{1}-a_{2}\right|$. Note that $w_{\lambda}\left(a_{1}, a_{2}\right)<\infty$ for all $a_{1}, a_{2} \in X$, then $X=X_{w}^{*}$ and $\left(X_{w}^{*}, w\right)$ is a complete modular metric space. Let $\Phi: R^{2} \longrightarrow R$ be given by $\Phi\left(t_{1}, t_{2}\right)=\max \left\{t_{1}, t_{2}\right\}$. Let $T: X_{w}^{*} \times X_{w}^{*} \longrightarrow X_{w}^{*}$ given by $T\left(a_{1}, a_{2}\right)=(32 / 1000)$ $\left(a_{1}+a_{2}\right)$. Then, 


$$
\begin{aligned}
w_{\xi \lambda}\left(T\left(a_{1}, a_{2}\right), T\left(a_{2}, a_{3}\right)\right) & =w_{\xi \lambda}\left(\frac{32}{1000}\left(a_{1}+a_{2}\right), \frac{32}{1000}\left(a_{2}+a_{3}\right)\right) \\
& =\frac{4}{15 \xi^{2} \lambda^{2}}\left|\frac{32}{1000}\left(a_{1}+a_{2}\right)-\frac{32}{100}\left(a_{2}+a_{3}\right)\right| \\
& =\frac{4}{15 \xi^{2} \lambda^{2}} \cdot \frac{32}{1000}\left|\left(a_{1}+a_{2}\right)-\left(a_{2}+a_{3}\right)\right| \\
& =\frac{4}{15 \xi^{2} \lambda^{2}} \cdot \frac{32}{1000}\left|\left(a_{1}-a_{2}\right)+\left(a_{2}-a_{3}\right)\right| \\
& =\frac{8}{15 \xi^{2} \lambda^{2}} \cdot \frac{32}{1000} \frac{\left|\left(a_{1}-a_{2}\right)+\left(a_{2}-a_{3}\right)\right|}{2} \\
& =\frac{2}{\xi^{2}} \cdot \frac{32}{1000} \frac{\left|\left(4 / 15 \lambda^{2}\right)\left(a_{1}-a_{2}\right)+\left(4 / 15 \lambda^{2}\right)\left(a_{2}-a_{3}\right)\right|}{2} \\
& \leq \frac{2}{\xi^{2}} \cdot \frac{32}{1000} \max \left\{w_{\lambda}\left(a_{1}, a_{2}\right), w_{\lambda}\left(a_{2}, a_{3}\right)\right\} .
\end{aligned}
$$

If $\xi=0.4 \in(0,(1 / 2))$, then $w_{0.4 \lambda}\left(T\left(a_{1}, a_{2}\right), T\left(a_{2}, a_{3}\right)\right) \leq$ $0.4 \Phi\left(w_{\lambda}\left(a_{1}, a_{2}\right), w_{\lambda}\left(a_{2}, a_{3}\right)\right)$ for all $a_{1}, a_{2}, a_{3} \in X_{w}$. Thus, $T$ is a generalised Presic type strongly modular contraction and Ciric-Presic type strongly modular contraction with constant $\xi=0.4$. Theorems 2 and 4 are applicable, and $(0,0) \in X_{w}$ is the unique fixed point of $T$.
Example 2. Let $X=[0,1]$. Define the mapping $w:(0, \infty) \times$ $X \times X \longrightarrow[0, \infty]$ by $w_{\lambda}\left(a_{1}, a_{2}\right)=\left(\left|a_{1}-a_{2}\right| / \lambda\right)$. Note that $w_{\lambda}\left(a_{1}, a_{2}\right)<\infty$ for all $a_{1}, a_{2} \in X$; then, $X=X_{w}^{*}$ and $\left(X_{w}^{*}, w\right)$ is a complete convex modular metric space. Let $\Phi: R^{3} \longrightarrow R$ be given by $\Phi\left(t_{1}, t_{2}, t_{3}\right)=t_{1}+t_{2}+t_{3}$, if $t_{i} \neq t_{j}$ for some $i, j$ and $\Phi(t, t, t)=t$.

Let $\quad T: X_{w}^{*} \times X_{w}^{*} \times X_{w}^{*} \longrightarrow X_{w}^{*}$ be given by $T\left(a_{1}, a_{2}, a_{3}\right)=(1 / 1.01)\left(a_{1}+a_{2}+a_{3}\right)$. Then,

$$
\begin{aligned}
w_{\xi \lambda}\left(T\left(a_{1}, a_{2}, a_{3}\right), T\left(a_{2}, a_{3}, a_{4}\right)\right) & =w_{\xi \lambda}\left(\frac{1}{1.01}\left(a_{1}+a_{2}+a_{3}\right), \frac{1}{1.01}\left(a_{2}+a_{3}+a_{4}\right)\right) \\
& =\frac{1}{\xi \lambda} \cdot \frac{1}{1.01}\left|\left(a_{1}+a_{2}+a_{3}\right)-\left(a_{2}+a_{3}+a_{4}\right)\right| \\
& =\frac{1}{\xi \lambda} \cdot \frac{1}{1.01}\left|\left(a_{1}-a_{2}\right)+\left(a_{2}-a_{3}\right)+\left(a_{3}-a_{4}\right)\right| .
\end{aligned}
$$

If $\xi=(1 / 1.01) \in(0,1)$, then $w_{(1 / 1.01) \lambda}\left(T\left(a_{1}, a_{2}, a_{3}\right)\right.$, $\left.T\left(a_{2}, a_{3}, a_{4}\right)\right) \leq \Phi\left(w_{\lambda}\left(a_{1}, a_{2}\right), w_{\lambda}\left(a_{2}, a_{3}\right), w_{\lambda}\left(a_{3}, a_{4}\right)\right)$ for all $a_{1}, a_{2}, a_{3}, a_{4} \in X_{w}$. Thus, $T$ is a generalised Presic type modular contraction, with constant $\xi=(1 / 1.01)$. Thus, $T$ satisfies the condition of Theorem 3 and $(0,0,0) \in X_{w}$ is the unique fixed point of $T$.

\section{Ulam-Hyers Stability}

In this section, we discuss the Ulam-Hyers stability of fixedpoint equations involving Presic type operators in a modular metric space. We begin with the following concepts and definitions.

Definition 5. Let $w$ be a metric modular on $X$ and $T: X_{w}^{k *} \longrightarrow X_{w}^{*}$ (where $X_{w}^{k *}$ denotes $X_{w}^{*} \times X_{w}^{*} \times \cdots k$ times) for some positive integer $k$. If for all $\left(x_{1}, x_{2}, \ldots, x_{k}\right) \in X_{w}^{k *}$ with $w_{\lambda}\left(x_{1}, x_{2}\right)<\infty, w_{\lambda}\left(x_{2}, x_{3}\right)<\infty, \ldots, w_{\lambda}\left(x_{k-1}, x_{k}\right)<\infty$, $w_{\lambda}\left(x_{k}, T\left(x_{1}, x_{2}, \ldots, x_{k}\right)\right)<\infty$, the sequence $x_{k+n}$ given by $x_{k+n}=T\left(x_{n}, x_{n+1}, \ldots, x_{k+n-1}\right)$ converges and the limit (which depends upon $\left.\left(x_{1}, x_{2}, \ldots, x_{k}\right)\right)$ is a fixed point of $T$, then we say that $T$ is a weakly Picard-Presic operator (in short, WPPO). If the fixed point of $T$ is unique, then we say that $T$ is a Picard-Presic operator.

Hereafter, we will make use of the following notations:

$$
\begin{aligned}
T^{n}\left(x_{1}, x_{2}, \ldots, x_{k}\right) & =x_{k+n}, \\
T^{\infty}\left(x_{1}, x_{2}, \ldots, x_{k}\right) & =\lim _{n \longrightarrow \infty} T^{n}\left(x_{1}, x_{2}, \ldots, x_{k}\right) \\
& =\lim _{n \longrightarrow \infty} x_{n+k} .
\end{aligned}
$$


Definition 6. Let $w$ be metric modular on $X$, $T: X_{w}^{k *} \longrightarrow X_{w}^{*}$ be a weakly Picard-Presic operator, and $c>0$ be a real number. We say that $T$ is a $c$-weakly
Picard-Presic operator (in short, $c$-WPPO) if for all $x=\left(x_{1}, x_{2}, \ldots, x_{k}\right) \in X_{w}^{k *}, 1 \leq i \leq k$, and for some $0<\xi<1$ and $\lambda_{0}>0$ possibly depending on $\xi$ and for all $0<\lambda \leq \lambda_{0}$,

$$
w_{\lambda}\left(x_{i}, T^{\infty}(x)\right) \leq c \max \left\{w_{\lambda}\left(x_{1}, x_{2}\right), w_{\lambda}\left(x_{2}, x_{3}\right), \ldots, w_{\lambda}\left(x_{k-1}, x_{k}\right), w_{\lambda}\left(x_{k}, T(x)\right)\right\}
$$

Let $R_{+}$be the set of nonnegative real numbers, and consider a function $\Psi: R_{+}^{k} \longrightarrow R_{+}$such that $\Psi$ is continuous at 0 and $\Psi(0)=0$.

Definition 7. Let $w$ be a metric modular on $X$; $T: X_{w}^{k *} \longrightarrow X_{w}^{*}$ be a weakly Picard-Presic operator. We say that $T$ is a $\Psi$-weakly Picard-Presic operator (in short, $\Psi-\mathrm{WPPO})$ if for all $x=\left(x_{1}, x_{2}, \ldots, x_{k}\right) \in X_{w}^{k *}, 1 \leq i \leq k$, and for some $0<\xi<1$ and $\lambda_{0}>0$ possibly depending on $\xi$ and for all $0<\lambda \leq \lambda_{0}$,

$$
w_{\lambda}\left(x_{i}, T^{\infty}(x)\right) \leq \Psi\left(\max \left\{w_{\lambda}\left(x_{1}, x_{2}\right), w_{\lambda}\left(x_{2}, x_{3}\right), \ldots, w_{\lambda}\left(x_{k-1}, x_{k}\right), w_{\lambda}\left(x_{k}, T(x)\right)\right\}\right)
$$

Proposition 1. Let T: $X_{w}^{k *} \longrightarrow X_{w}^{*}$ be a Ciric-Presic strong $w$-contraction. Then, $T$ is a $c-W P P O$.

$$
\begin{aligned}
w_{\lambda}\left(x_{i}, T^{n}(x)\right) \leq & w_{\left(\xi^{m+1}+\xi^{m+2}+\cdots \xi^{m+n+1}\right) \lambda}\left(x_{i}, T^{n}(x)\right) \\
\leq & w_{\xi^{m+1} \lambda}\left(x_{i}, T(x)\right)+w_{\xi^{m+2} \lambda}\left(T(x), T^{2}(x)\right)+\cdots w_{\xi^{m+n+1} \lambda}\left(T^{n-1}(x), T^{n}(x)\right) \\
\leq & w_{\left(\xi^{r}+\xi^{r+1}+\cdots \xi^{\xi^{+k-i}}\right) \lambda}\left(x_{i}, T(x)\right)+w_{\xi^{2} \lambda}\left(T(x), T^{2}(x)\right)+\cdots w_{\xi^{n+1} \lambda}\left(T^{n-1}(x), T^{n}(x)\right) \\
\leq & \left.w_{\left(\xi^{r}+\xi^{r^{+1}}+\cdots \xi^{\xi^{+k-i}}\right) \lambda}\left(x_{i}, x_{k+1}\right)+w_{\xi^{2} \lambda}\left(x_{k+1}, x_{k+2}\right)\right)+\cdots w_{\xi^{n+1} \lambda}\left(x_{n-1}, x^{n}\right) \\
\leq & w_{\left(\xi^{r} \lambda\right.}\left(x_{i}, x_{i+1}\right)+w_{\left(\xi^{r+1} \lambda\right.}\left(x_{i+1}, x_{i+2}\right)+\cdots w_{\left(\xi^{r+k-i-1} \lambda\right.}\left(x_{k-1}, x_{k}\right)+w_{\left(\xi^{r+k-i} \lambda\right.}\left(x_{k}, x_{k+1}\right) \\
& \left.\quad+w_{\xi^{2} \lambda}\left(x_{k+1}, x_{k+2}\right)\right)+\cdots w_{\xi^{n+1} \lambda}\left(x_{n-1}, x^{n}\right) .
\end{aligned}
$$

Using (15), we get

$$
w_{\lambda}\left(x_{i}, T^{n}(x)\right) \leq R_{\xi \lambda}\left[\xi^{(i / k)}+\xi^{(i+1 / k)}+\cdots \xi^{(k-1 / k)}+\xi^{(k / k)}+\cdots \xi^{(n-1 / k)}\right] .
$$


As $n \longrightarrow \infty$, we get

$$
\begin{aligned}
w_{\lambda}\left(x_{i}, T^{\infty}(x)\right) & \leq R_{\xi \lambda}\left[1+\xi^{(i / k)}+\xi^{(i+1 / k)}+\ldots \xi^{(k-1 / k)}+\xi^{(k / k)}+\cdots\right] \\
& \leq \frac{1}{1-\xi^{(1 / k)}} \max \left(\frac{\alpha_{1_{\lambda}}}{\theta}, \frac{\alpha_{2_{\lambda}}}{\theta^{2}}, \ldots, \frac{\alpha_{k_{\lambda}}}{\theta^{k}}\right) \\
& \leq \frac{1}{1-\xi^{(1 / k)}} \max \left(\frac{w_{\lambda}\left(x_{1}, x_{2}\right)}{\xi^{(1 / k)}}, \frac{w_{\lambda}\left(x_{2}, x_{3}\right)}{\xi^{(2 / k)}}, \ldots, \frac{w_{\lambda}\left(x_{k-1}, x_{k}\right)}{\xi^{(k-1 / k)}}, \frac{w_{\lambda}\left(x_{k}, T(x)\right)}{\xi^{(k / k)}}\right) \\
& \leq \frac{1}{\xi\left(1-\xi^{(1 / k)}\right)} \max \left(w_{\lambda}\left(x_{1}, x_{2}\right), w_{\lambda}\left(x_{2}, x_{3}\right), \ldots, w_{\lambda}\left(x_{k-1}, x_{k}\right), w_{\lambda}\left(x_{k}, T(x)\right)\right) .
\end{aligned}
$$

Hence, $T$ is a $c-$ WPPO with $c=\left(1 / \xi\left(1-\xi^{(1 / k)}\right)\right)$.

For some $x^{*} \in \operatorname{Fix}\left(X_{w}^{*}\right)$, the attraction basin of $x^{*}$ with respect to $T$ is given by

$$
(A B)_{T}\left(x^{*}\right)=\left\{x=\left(x_{1}, x_{2}, \ldots, x_{k}\right) \in X_{w}^{k *}: T^{n}(x) \text { is defined for all } n \in N \text { and } T^{n}(x) \longrightarrow x^{*}\right\}
$$

and the attraction basin of $T$ is given by

$$
(A B)_{T}=\bigcup_{x^{*} \in \operatorname{Fix}(T)}(A B)_{T}\left(x^{*}\right) \text {. }
$$

For some $T: X_{w}^{k *} \longrightarrow X_{w}^{*}$, we consider the fixed-point equation

$$
x=T(x, x, \ldots, x), \quad x \in X_{w}^{*},
$$

and the inequality

$$
w_{\lambda}(y, T(y, y, \ldots, y) \leq \epsilon, \quad \text { for all } \lambda>0 .
$$

Definition 8. Equation (39) is Ulam-Hyers stable if there exists $c>0$ such that for each $\epsilon>0$ and each solution $y^{*}$ of (40) with $\left(y^{*}, y^{*}, \ldots, y^{*}\right) \in(A B)_{T}$, there exists a solution $x^{*}$ of fixed-point equation (39) such that $w_{\lambda}\left(y^{*}, x^{*}\right) \leq c \epsilon$ for all $\lambda>0$.
Definition 9. Equation (39) is generalised Ulam-Hyers stable if there exists a function $\phi: R^{+} \longrightarrow R^{+}$increasing, continuous at 0 and $\phi(0)=0$, such that for each $\epsilon>0$ and each solution $y^{*}$ of $(40)$ with $\left(y^{*}, y^{*}, \ldots, y^{*}\right) \in(A B)_{T}$, there exists a solution $x^{*}$ of fixed-point equation (39) such that $w_{\lambda}\left(y^{*}, x^{*}\right) \leq \phi(\epsilon)$ for all $\lambda>0$.

Theorem 7. Let $w$ be strict metric modular on $X$ and $\left(X_{w}^{*}, w\right)$ be $w$-complete. For any positive integer $k$, let $T: X_{w}^{k *} \longrightarrow X_{w}^{*}$ be a $c-W P P O$. Then, fixed-point equation (39) is Ulam-Hyers stable.

Proof. Let $\epsilon>0, \lambda>0$, and $y^{*}$ be a solution of (39) with $y=\left(y^{*}, y^{*}, \ldots, y^{*}\right) \in(A B)_{T} ;$ that is, $w_{\lambda}\left(y^{*}, T(y)\right) \leq \epsilon$. Then, there exists $x^{*} \in \operatorname{Fix}(T)$; that is, $x^{*}$ is a solution of (39) such that $T^{n}(y) \longrightarrow x^{*}$. Since $T: X_{w}^{k *} \longrightarrow X_{w}^{*}$ is a $c$ - WPPO, we have

$$
\begin{aligned}
w_{\lambda}\left(y^{*}, T^{\infty}(y)\right) & \leq c \cdot \max \left\{w_{\lambda}\left(y^{*}, y^{*}\right), w_{\lambda}\left(y^{*}, y^{*}\right), \ldots, w_{\lambda}\left(y^{*}, y^{*}\right), w_{\lambda}\left(y^{*}, T(y)\right)\right\} \\
& \leq c \epsilon .
\end{aligned}
$$
stable.

Thus, fixed-point equation (39) is Ulam-Hyers

Remark 4. For $k=1$, Definitions 6-9 reduce to Definitions $1,2,7$, and 8 , respectively, of [30].

\section{Application to Integral Equation}

Recently, some interesting applications of fixed-point theorems in proving the existence of solutions of various generalised integral equations and differential equations have been found (see [31-33]. Moreover, in [33], the Ulam-Hyers stability of nonlinear implicit fractional differential equations with Riemann-Liouville fractional derivative was discussed. In this section, we present an application of our results to prove the existence of solution of a generalised integral equation of Caratheodory type and give the conditions under which such integral equations are Ulam-Hyers stable. Let $\phi: R^{+} \longrightarrow R^{+}$be a continuous, convex, increasing, and unbounded function such that 
$\phi(u)=0$ iff $u=0$. Moreover, $\phi$ admits the inverse function $\phi^{-1}: R^{+} \longrightarrow R^{+}$, which is continuous and strictly increasing, and $\phi^{-1}(u)=0$ iff $u=0$. Let $\mathfrak{M}$ be a set of real-valued function on $[a, b] \subset R$ with $a<b$; that is,
$\mathfrak{M}=\{\mathfrak{t}:[a, b] \longrightarrow \mathfrak{A}\}, \quad \mathfrak{A} \subset R$ is complete.

Define $w:(0, \infty) \times \mathfrak{M} \times \mathfrak{M} \longrightarrow[0, \infty]$ for all $\gamma>0$ and $\mathfrak{u}, \mathfrak{w} \in \mathfrak{M}$ by

$$
w_{\gamma}(\mathfrak{t}, \mathfrak{w})=\sup _{\pi} \sum_{i=1}^{m} \phi\left(\frac{\left|\left[\mathfrak{t}\left(\tau_{i}\right)+\mathfrak{w}\left(\tau_{i-1}\right)\right]-\left[\mathfrak{t}\left(\tau_{i-1}\right)+\mathfrak{w}\left(\tau_{i}\right)\right]\right|}{\gamma\left(\tau_{i}-\tau_{i-1}\right)}\right)\left(\tau_{i}-\tau_{i-1}\right),
$$

where the supremum is taken over all partitions $\pi=\left\{\tau_{i}\right\}_{i=0}^{n}$ of the interval $[a, b]$; that is, $a=\tau_{0}<\tau_{1}<\tau_{2} \cdots<\tau_{n}=b$. Then, it is known that $w_{\gamma}(\mathfrak{u}, \mathfrak{w})$ is convex pseudomodular on $\mathfrak{M}($ see $[9,10])$.
For some $u_{0} \in R$, consider the constant function $\mathfrak{u}_{0} \in \mathfrak{M}$ given by $\mathfrak{t}_{0}(\tau)=u_{0}$ for all $\tau \in[a, b]$. Define the convex pseudomodular metric space $\mathfrak{M}_{w}^{*}$ as

$$
\mathfrak{M}_{w}^{*}=\mathfrak{M}_{w}^{*}\left(\mathfrak{t}_{0}\right)=\left\{\mathfrak{u} \in \mathfrak{M}: \exists \gamma=\gamma(\mathfrak{t})>0 \text {, such that } w_{\gamma}\left(\mathfrak{u}, \mathfrak{t}_{0}\right)<\infty\right\}
$$

The space $\mathfrak{M}_{w}^{*}$ is denoted by $G V_{\phi}([a, b])$ and is called the space of mappings of bounded generalised $\phi$ - variations (see [34]). Then, $\mathfrak{u} \in \mathfrak{M}_{w}^{*}=G V_{\phi}([a, b])$ if and only if $\mathfrak{t}:[a, b] \longrightarrow R$, and there exists a constant $\gamma=\gamma(\mathfrak{u})>0$ such that

$$
w_{\gamma}\left(\mathfrak{t}, \mathfrak{u}_{0}\right)=\sup _{\pi} \sum_{i=1}^{n} \phi\left(\frac{\left|\mathfrak{u}\left(\tau_{i}\right)-\mathfrak{t}\left(\tau_{i-1}\right)\right|}{\gamma\left(\tau_{i}-\tau_{i-1}\right)}\right)\left(\tau_{i}-\tau_{i-1}\right)<\infty .
$$

Clearly, $w\left(\gamma, \mathfrak{t}, \mathfrak{t}_{0}\right)$ is independent of. $\mathfrak{t}_{0}$

Consider the set $\mathfrak{M}^{1}$ given by

$$
\mathfrak{M}^{1}=\left\{\mathfrak{u} ; \mathfrak{u}:[a, b] \longrightarrow \mathfrak{A}, \mathfrak{u}(a)=u_{0}\right\} \subset \mathfrak{M}_{w}^{*} \text {. }
$$

Lemma 1. The function $w$ given in (43) defines a strict convex metric modular on $\mathfrak{M}^{2}$.

Proof. It is enough to show that $w_{\gamma}(\mathfrak{t}, \mathfrak{w})=0 \Longrightarrow \mathfrak{u}(\tau)=$ $\mathfrak{w}(\tau)$ for all $\mathfrak{t}, \mathfrak{w} \in \mathfrak{M}^{1}$ and $\tau \in[a, b]$. Clearly, for any $\psi, \tau \in[a, b]$, with $\psi \neq \tau$,

$$
\phi\left(\frac{|[\mathfrak{u}(\tau)+\mathfrak{w}(\psi)]-[\mathfrak{u}(\psi)+\mathfrak{w}(\tau)]|}{\gamma|\tau-\psi|}\right)(\tau-\psi) \leq w_{\gamma}(\mathfrak{u}, \mathfrak{w}),
$$

which implies

$$
|\mathfrak{u}(\tau)-\mathfrak{w}(\tau)+\mathfrak{w}(\psi)-\mathfrak{u}(\psi)| \leq \gamma|\tau-\psi| \phi^{-1}\left(\frac{w_{\gamma}(\mathfrak{t}, \mathfrak{w})}{\tau-\psi}\right) .
$$

Let $w_{\gamma}(\mathfrak{h}, \mathfrak{w})=0$. Then, since $\phi^{-1}(0)=0$, we get

$$
|\mathfrak{u}(\tau)-\mathfrak{w}(\tau)+\mathfrak{w}(\psi)-\mathfrak{u}(\psi)| \leq \gamma|\tau-\psi| \phi^{-1}(0)=0 .
$$

Equivalently,

$$
\mathfrak{u}(\tau)-\mathfrak{w}(\tau)=\mathfrak{t}(\psi)-\mathfrak{w}(\psi) .
$$

Solving the system by taking $\psi=a$ in (50) and using $\mathfrak{u}(a)=\mathfrak{w}(a)=u_{0}$, we obtain $\mathfrak{t}(\tau)=\mathfrak{w}(\tau)$ for any $\tau \in[a, b]$.

We define

$$
\begin{aligned}
\mathfrak{M}_{w}^{* 1} & =M_{w}^{*} \cap \mathfrak{M}^{1}=G V_{\phi}([a, b]) \cap \mathfrak{M}^{1}=\left\{\mathfrak{u} \in G V_{\phi}([a, b]), \mathfrak{u}(a)=u_{0}\right\} \\
& =\left\{\mathfrak{u} \in \mathfrak{M}^{1}: \exists \gamma=\gamma(\mathfrak{u})>0, \text { such that } w_{\gamma}\left(\mathfrak{u}, \mathfrak{u}_{0}\right)<\infty\right\}
\end{aligned}
$$

Then, $\mathfrak{M}_{w}^{* 1}$ is a metric modular space.

Lemma 2. The metric modular space $\mathfrak{M}_{w}^{* 1}$ is $w$-complete.

Proof. Let $\left\{\mathfrak{u}_{n}\right\} \subset \mathfrak{M}_{w}^{* 1}$ be $w$-Cauchy. Then,

$$
w_{\gamma}\left(\mathfrak{t}_{n}, \mathfrak{u}_{m}\right) \longrightarrow 0, \quad \text { as } n, m \longrightarrow \infty,
$$

for some $\gamma=\gamma\left(\left\{\mathfrak{u}_{n}\right\}\right)>0$. Therefore, for $n, m \in N$ and $t \in[a, b]$, we have

$$
\begin{aligned}
\left|\mathfrak{u}_{n}(\tau)-\mathfrak{t}_{m}(\tau)\right| & =\left|\left[\mathfrak{u}_{n}(\tau)-\mathfrak{t}_{m}(\tau)\right]-\mathfrak{t}_{n}(0)+\mathfrak{t}_{m}(0)\right| \\
& \leq \mid\left[\mathfrak{t}_{n}(\tau)+\mathfrak{t}_{m}(0)\right]-\left[\mathfrak{u}_{m}(\tau)+\mathfrak{t}_{n}(0)\right] \\
& \leq \gamma|t-0| \phi^{-1} \frac{w_{\gamma}\left(\mathfrak{t}_{n}, \mathfrak{t}_{m}\right)}{(t-0)} \\
& \longrightarrow 0, \quad \text { as } n, m \longrightarrow \infty
\end{aligned}
$$


which implies that $\operatorname{Lim}_{n, m \rightarrow \infty}\left|\mathfrak{t}_{n}(\tau)-\mathfrak{t}_{m}(\tau)\right|=0$. Since $\mathfrak{A}$ is complete, the sequence $\left\{\mathfrak{u}_{n}\right\}$ converges to some $\mathfrak{t}:[a, b] \longrightarrow \mathfrak{A}$ with $\mathfrak{t}(a)=u_{0}$. That is, for all $t \in[a, b]$, $\operatorname{Lim}_{n \longrightarrow \infty}\left|\mathfrak{t}_{n}(\tau)-\mathfrak{t}_{m}(\tau)\right|=0$ holds for $\mathfrak{u} \in \mathfrak{M}^{1}$. It remains to show that $\operatorname{Lim}_{n \longrightarrow \infty} w_{\gamma}\left(\mathfrak{u}_{n}(\tau), \mathfrak{u}(\tau)\right)=0$. From lower semicontinuity of $w$ (see [9], p.27), we have

$$
w_{\gamma}\left(\mathfrak{t}_{n}, \mathfrak{t}\right) \leq \lim _{n \longrightarrow \infty} \inf w_{\gamma}\left(\mathfrak{t}_{n}, \mathfrak{t}_{m}\right),
$$

for every $n \in N$. Since $\left\{\mathfrak{u}_{n}\right\}$ is $w$-Cauchy, for all $\epsilon>0$, there exists $N(\epsilon) \in N$ such that $\forall n, m \geq N(\epsilon), w_{\gamma}\left(\mathfrak{u}_{n}, \mathfrak{u}_{m}\right)<\epsilon$.

Hence, for all $n \geq N(\epsilon)$,

$$
\lim _{m \longrightarrow \infty} \sup w_{\gamma}\left(\mathfrak{u}_{n}, \mathfrak{t}_{m}\right) \leq \sup _{m \geq N(\epsilon)} w_{\gamma}\left(\mathfrak{t}_{n}, \mathfrak{t}_{m}\right)<\epsilon .
$$

Thus, for every $\epsilon>0$, there exists $N(\epsilon) \in N$ such that

$$
w_{\gamma}\left(\mathfrak{u}_{n}, \mathfrak{t}\right) \leq \operatorname{Lim}_{m \longrightarrow \infty} \inf w\left(\gamma, \mathfrak{t}_{n}, \mathfrak{u}_{m}\right) \leq \operatorname{Lim}_{m \longrightarrow \infty} \sup w\left(\gamma, \mathfrak{t}_{n}, \mathfrak{t}_{m}\right)<\epsilon
$$

Then, $\left\{\mathfrak{u}_{n}\right\}$ is $w$-convergent to $\mathfrak{t}$. As $\mathfrak{M}_{w}^{* 1}$ is closed under the modular convergence, we have $\mathfrak{t} \in \mathfrak{M}_{w}^{* 1}$, and thus, $\mathfrak{M}_{w}^{* 1}$ is $w$-complete.
Furthermore, if $\phi$ satisfies the Orlicz condition at infinity, that is, $(\phi(\mathfrak{w}) / \mathfrak{w}) \longrightarrow \infty$ as $\mathfrak{w} \longrightarrow \infty$, then $w_{1}(\mathfrak{u}, 0)$ is called the $\phi$-variation of the function $\mathfrak{t}:[a, b] \longrightarrow R$; the function $\mathfrak{t}$ with $w_{1}(\mathfrak{u}, 0)<\infty$ is said to be of bounded $\phi$-variation on $[a, b]$ and we have

$$
w_{\gamma}(\mathfrak{u}, \mathfrak{w})=w_{\gamma}(\mathfrak{u}-\mathfrak{w}, 0)=w_{1}\left(\frac{\mathfrak{u}-\mathfrak{w}}{\gamma}, 0\right), \quad \gamma>0, \mathfrak{t}, \mathfrak{w} \in \mathfrak{M} .
$$

For the functions $\mathfrak{t}:[a, b] \longrightarrow \mathfrak{A}$ in the space $\mathfrak{M}_{w}^{*}=G V_{\phi}([a, b])$, it is known that (see $\left.[10,35]\right)$

$$
\begin{aligned}
\mathfrak{u} \in G V_{\phi}([a, b]) & \Leftrightarrow w_{\gamma}(\mathfrak{t}, 0)<\infty, \quad \text { for some } \gamma=\gamma(\mathfrak{t})>0, \\
& \Leftrightarrow \mathfrak{u} \in A C[a, b], \mathfrak{t}^{\prime} \in L^{1}[a, b] \text { with } w_{\gamma}(\mathfrak{t}, 0)=\int_{a}^{b} \phi\left(\frac{\left|\mathfrak{t}^{\prime}(\tau)\right|}{\gamma}\right) \mathrm{d} \tau<\infty,
\end{aligned}
$$

where $A C[a, b]$ is the space of all absolutely continuous realvalued functions on $[a, b]$ and $L^{1}[a, b]$ is the space of all Lebesgue integrable functions on $[a, b]$.

Now, we apply the result given in Theorem 2 to the following integral equation:

$$
\mathfrak{t}(\psi)=\int_{a}^{\mu(\psi)} K(\psi, v, \mathfrak{u}(v)) \mathrm{d} v+\int_{a}^{\sigma(\psi)} J(\psi, v, \mathfrak{u}(v)) \mathrm{d} v+\mathfrak{w}(\psi),
$$

for $\quad \psi \in[a, b], \quad$ where $\mathfrak{u}, \mathfrak{w} \in \mathfrak{M}_{w}^{* 1}, \quad K, J:[a, b] \times[a, b]$ $\times \mathfrak{A} \longrightarrow R$ are Caratheodory type functions, $\mu, \sigma:[a, b]$ $\longrightarrow[a, b]$, and $\mu(a)=\sigma(a)=a$. Define the function $T: \mathfrak{M}_{w}^{* 1} \times \mathfrak{M}_{w}^{* 1} \longrightarrow \mathfrak{M}_{w}^{* 1}$ by

$$
T\left(\mathfrak{t}_{1}, \mathfrak{u}_{2}\right)(\psi)=\int_{a}^{\mu(\psi)} K\left(\psi, v, \mathfrak{t}_{1}(v)\right) \mathrm{d} v+\int_{a}^{\sigma(\psi)} J\left(\psi, v, \mathfrak{t}_{2}(v)\right) \mathrm{d} v+\mathfrak{w}(\psi),
$$

where $\mathfrak{t}_{1}, \mathfrak{t}_{2}, \mathfrak{w} \in \mathfrak{M}_{w}^{* 1}$. Then, problem (60) is equal to the fixed-point problem:

$T(u, u)=u$ 
Definition 10. Equation (60) is Ulam-Hyers stable, if there exists $c>0$ such that for each $\epsilon>0$ and $u^{*} \in \mathfrak{M}_{w}^{* 1}$ for which

$$
\sup _{\pi} \sum_{i=1}^{m} \phi\left(\frac{\mid\left[u^{*}\left(\tau_{i}\right)+T\left(u^{*}, u^{*}\right)\left(\tau_{i-1}\right]-\left[u^{*}\left(\tau_{i-1}\right)+T\left(u^{*}, u^{*}\right)\left(\tau_{i}\right] \mid\right.\right.}{\gamma\left(\tau_{i}-\tau_{i-1}\right)}\right)\left(\tau_{i}-\tau_{i-1}\right) \leq \epsilon,
$$

there exists a solution $u$ of (60) such that $\sup _{\pi} \sum_{i=1}^{m} \phi\left(\left|\left[u^{*}\left(\tau_{i}\right)+u\left(\tau_{i-1}\right)\right]-\left[u^{*}\left(\tau_{i-1}\right)+u\left(\tau_{i}\right)\right]\right| / \gamma\left(\tau_{i}-\right.\right.$ $\left.\left.\tau_{i-1}\right)\right)\left(\tau_{i}-\tau_{i-1}\right) \leq c \epsilon$, for all $\tau \in[a, b]$, where the supremum is taken over all partitions $\pi=\left\{\tau_{i}\right\}_{i=0}^{n}$ of the interval $[a, b]$; that is, $a=\tau_{0}<\tau_{1}<\tau_{2} \cdots<\tau_{n}=b$ and $\phi: R^{+} \longrightarrow R^{+}$is a continuous, convex, increasing, and unbounded function such that $\phi(u)=0$ iff $u=0$ admits the inverse function $\phi^{-1}: R^{+} \longrightarrow R^{+}$, which is continuous and strictly increasing, and $\phi^{-1}(u)=0$ iff $u=0$.

If $\phi$ also satisfies Orlicz condition at infinity, then the above definition is equivalent to the following.

Definition 11. Equation (60) is Ulam-Hyers stable if there exists $c>0$ such that for each $\epsilon>0$ and $u^{*} \in \mathfrak{M}_{w}^{* 1}$ for which $\int_{a}^{b} \phi\left(\mid u^{* \prime}(\tau)-K\left(\tau, v, u^{*}(\mu(\tau))-K\left(\tau, v, u^{*}(\sigma(\tau))-u^{\prime}(\tau) \mid /\right.\right.\right.$ $\gamma) \mathrm{d} \tau \leq \epsilon$ there exists a solution $u$ of (60) such that $\int_{a}^{b} \phi\left(\left|u^{* \prime}(\tau)-u^{\prime}(\tau)\right| / \gamma\right) \mathrm{d} \tau \leq c \epsilon$ for all $\tau \in[a, b]$.
We will analyse problem (60) under the following conditions:

(m1) For every $\mathfrak{u}_{j} \in R$, where $j \in N$, the functions $K\left(\psi, v, \mathfrak{u}_{j}\right)$ and $J\left(\psi, v, \mathfrak{t}_{j}\right)$ are Lebesgue measurable on $[a, b]$ and there exist points $u_{0}, v_{0} \in R$ such that

$$
\begin{gathered}
\int_{a}^{b} \phi\left(\frac{\left|K\left(\psi, v, u_{0}\right)\right|}{\gamma_{K}}\right) \mathrm{d} v<\infty, \\
\int_{a}^{b} \phi\left(\frac{\left|J\left(\psi, v, v_{0}\right)\right|}{\gamma_{J}}\right) \mathrm{d} v<\infty,
\end{gathered}
$$

for some $\gamma_{K}=\gamma\left(K\left(\psi, v, u_{0}\right)\right)>0$ and $\gamma_{J}=$ $\gamma\left(J\left(\psi, v, v_{0},\right)\right)>0$, where $\phi$ is a function satisfying the Orlicz condition at infinity and $\gamma>0$.

(m2) There exists a constant $L>0$ such that

$$
\left|K\left(\psi, v, \mathfrak{t}_{1}\right)-K\left(\psi, v, \mathfrak{u}_{2}\right)\right|+\left|J\left(\psi, v, \mathfrak{u}_{2}\right)-J\left(\psi, v, \mathfrak{u}_{3}\right)\right| \leq L^{2}(b-a) \max \left\{\left|\mathfrak{u}_{1}-\mathfrak{u}_{2}\right|,\left|\mathfrak{u}_{2}-\mathfrak{u}_{3}\right|\right\}
$$

for almost all $\psi, v \in[a, b]$ and $\mathfrak{t}_{1}, \mathfrak{t}_{2} \in \mathfrak{A}$.

$$
w_{L(b-a) \gamma}\left(T\left(\mathfrak{t}_{1}, \mathfrak{t}_{2}\right), T\left(\mathfrak{t}_{2}, \mathfrak{t}_{3}\right)\right) \leq L(b-a) \max \left\{w_{\gamma}\left(\mathfrak{t}_{1}, \mathfrak{t}_{2}\right), w_{\gamma}\left(\mathfrak{t}_{2}, \mathfrak{t}_{3}\right)\right\},
$$

$$
\phi(a \mathfrak{u}(\tau)) \leq a \phi(\mathfrak{t}(\tau)), \quad 0<a<1, \mathfrak{u}(\tau) \in R,
$$

for all $\gamma>0$ and $\mathfrak{t}_{1}, \mathfrak{t}_{2}, \mathfrak{t}_{3} \in \mathfrak{M}_{w}^{* 1}$.

Proof. Applying Jensen's integral inequality with the convex function $\phi$,

$$
\phi\left(\frac{1}{b-a} \int_{a}^{b}|\mathfrak{t}(\tau)| \mathrm{d} \tau\right) \leq \frac{1}{b-a} \int_{a}^{b} \phi(|\mathfrak{u t}(\tau)|) \mathrm{d} \tau, \quad \mathfrak{t} \in L^{1}[a, b],
$$

and by the property of the convex function $\phi$, where the integral on the RHS takes values in $[0, \infty]$.

Step 1. Claim: $T$ is well defined on $\mathfrak{M}_{w}^{* 1} \times \mathfrak{M}_{w}^{* 1}$.

Let $\quad \mathfrak{t}_{1}, \mathfrak{t}_{2} \in \mathfrak{M}_{w}^{* 1}$, i.e., $\quad \mathfrak{t}_{1}, \mathfrak{t}_{2} \in G V_{\phi}[a, b] \quad$ and $\mathfrak{t}_{1}(a)=\mathfrak{t}_{2}(a)=\mathfrak{t}_{0}$. Since $\mathfrak{t}_{1}, \mathfrak{t}_{2} \in A C[a, b]$, in the light of (m1) and (m2), the functions $K$ and $J$ are measurable on $[a, b]$. Let us prove that $K, J \in L^{1}[a, b]$. By Lebesgue's theorem, $\mathfrak{t}(v)=\mathfrak{t}_{0}+\int_{a}^{v} \mathfrak{t t}^{\prime}(\psi) \mathrm{d} \psi$ for all $v \in[a, b]$ and so, by (m2), we have 


$$
\begin{aligned}
& \left|K\left(\psi, v, \mathfrak{u}_{1}(v)\right)\right|+\left|J\left(\psi, v, \mathfrak{t}_{2}(v)\right)\right| \\
& \quad \leq\left|K\left(\psi, v, \mathfrak{u}_{1}(v)\right)-K\left(\psi, v, u_{0}\right)\right|+\left|K\left(\psi, v, u_{0}\right)\right|+\left|J\left(\psi, v, \mathfrak{u}_{2}(v)\right)-J\left(\psi, v, v_{0}\right)\right|+\left|J\left(\psi, v, v_{0}\right)\right| \\
& \quad \leq L^{2}(b-a) \max \left\{\left|\mathfrak{u}_{1}(v)-\mathfrak{u}_{0}\right|,\left|\mathfrak{t}_{2}(v)-v_{0}\right|\right\}+\left|K\left(\psi, v, u_{0}\right)\right|+\left|J\left(\psi, v, v_{0}\right)\right| \\
& \quad \leq L^{2}(b-a) \max \left\{\int_{a}^{b} \mathfrak{t}_{1}^{\prime}(v) \mathrm{d} v+\left|\mathfrak{u}_{0}-u_{0}\right|, \int_{a}^{b} \mathfrak{t}_{2}^{\prime}(v) \mathrm{d} v+\left|\mathfrak{t}_{0}-v_{0}\right|\right\}+K\left(\psi, v, u_{0}\right)+J\left(\psi, v, v_{0}\right) .
\end{aligned}
$$

Therefore,

$$
\begin{aligned}
\left|K\left(\psi, v, \mathfrak{u}_{1}(v)\right)\right|+\left|J\left(\psi, v, \mathfrak{u}_{2}(v)\right)\right| \leq & L^{2}(b-a) \max \left\{\int_{a}^{b} \mathfrak{u}_{1}^{\prime}(v) \mathrm{d} v+\left|\mathfrak{u}_{0}-u_{0}\right|,\right. \\
& \left.\int_{a}^{b} \mathfrak{u}_{2}^{\prime}(v) \mathrm{d} v+\left|\mathfrak{u}_{0}-v_{0}\right|\right\}+\left|K\left(\psi, v, u_{0}\right)\right|+\left|J\left(\psi, v, v_{0}\right)\right|,
\end{aligned}
$$

for almost all $v \in[a, b]$. Since $\mathfrak{u}_{1}, \mathfrak{t}_{2} \in \mathfrak{M}_{w}^{* 1}$, we have $\mathfrak{u}_{1}, \mathfrak{t}_{2} \in G V_{\phi}[a, b]$ and so there exist constants $\gamma_{1}=\gamma_{1}\left(\mathfrak{t}_{1}\right)>0$ and $\gamma_{2}=\gamma_{2}\left(\mathfrak{t}_{2}\right)>0$ such that

$$
\begin{aligned}
& C_{1} \equiv w_{\gamma_{1}}\left(\mathfrak{u}_{1}, \mathfrak{t}_{0}\right)=\int_{a}^{b} \phi\left(\frac{\left|\mathfrak{u}_{1}^{\prime}(v)\right|}{\gamma_{1}}\right) \mathrm{d} v<\infty, \\
& C_{1}^{\prime} \equiv w_{\gamma_{2}}\left(\mathfrak{u}_{2}, \mathfrak{u}_{0}\right)=\int_{a}^{b} \phi\left(\frac{\left|\mathfrak{u}_{2}^{\prime}(v)\right|}{\gamma_{2}}\right) \mathrm{d} v<\infty .
\end{aligned}
$$

Also by (m1), there exist constants $\gamma_{K}=\gamma\left(K\left(\psi, v, v_{0}\right)\right)>0$ and $\gamma_{J}=\gamma\left(J\left(\psi, v, v_{0}\right)\right)>0$ such that

$$
\begin{aligned}
& C_{2}=\int_{a}^{b} \phi\left(\frac{\left|K\left(\psi, v, u_{0}\right)\right|}{\gamma_{K}}\right) \mathrm{d} v<\infty, \\
& C_{3}=\int_{a}^{b} \phi\left(\frac{\left|J\left(\psi, v, v_{0}\right)\right|}{\gamma_{J}}\right) \mathrm{d} v<\infty .
\end{aligned}
$$

If $\max \left\{\int_{a}^{b} \mathfrak{u}_{1}^{\prime}(v) \mathrm{d} v+\left|\mathfrak{u}_{0}-u_{0}\right|, \int_{a}^{b} \mathfrak{u}_{2}^{\prime}(v) \mathrm{d} v+\left|\mathfrak{u}_{0}-v_{0}\right|\right\}=$ $\int_{a}^{b} \mathfrak{t}_{1}^{\prime}(v) \mathrm{d} v+\left|\mathfrak{u}_{0}-u_{0}\right|$ by the convexity of $\phi$, we find

$$
\begin{aligned}
\phi( & \left.\frac{\left|K\left(\psi, v, \mathfrak{t}_{1}(v)\right)\right|+\left|J\left(\psi, v, \mathfrak{t}_{2}(v)\right)\right|}{\gamma_{0}}\right) \\
& \leq \phi\left(\frac{1}{\gamma_{0}}\left[L^{2}(b-a) \int_{a}^{b} \mathfrak{t}_{1}^{\prime}(v) \mathrm{d} v+L^{2}(b-a)\left|\mathfrak{t}_{0}-u_{0}\right|+\left|K\left(\psi, v, u_{0}\right)\right|+\left|J\left(\psi, v, v_{0}\right)\right|\right]\right) \\
& =\phi\left(\frac{L^{2}(b-a) \gamma_{1}}{\gamma_{0}} \int_{a}^{b} \frac{\mathfrak{t}_{1}^{\prime}(v)}{\gamma_{1}} \mathrm{~d} \psi+\frac{1}{\gamma_{0}} L^{2}(b-a)\left|\mathfrak{t}_{0}-u_{0}\right|+\frac{\gamma_{K}}{\gamma_{0}} \frac{\left|K\left(\psi, v, u_{0}\right)\right|}{\gamma_{K}}+\frac{\gamma_{J}}{\gamma_{0}} \frac{\left|J\left(\psi, v, v_{0}\right)\right|}{\gamma_{J}}\right) \\
& \leq \frac{L^{2}(b-a) \gamma_{1}}{\gamma_{0}} \phi\left(\int_{a}^{b} \frac{\mathfrak{u}_{1}^{\prime}(v)}{\gamma_{1}} \mathrm{~d} \psi\right)+\frac{1}{\gamma_{0}} \phi\left(L^{2}(b-a)\left|\mathfrak{t}_{0}-u_{0}\right|\right)+\frac{\gamma_{K}}{\gamma_{0}} \phi\left(\frac{\left|K\left(\psi, v, u_{0}\right)\right|}{\gamma_{K}}\right)+\frac{\gamma_{J}}{\gamma_{0}} \phi\left(\frac{\left|J\left(\psi, v, v_{0}\right)\right|}{\gamma_{J}}\right) \\
& =\frac{L^{2}(b-a) \gamma_{1}}{\gamma_{0}} C_{1}+\frac{1}{\gamma_{0}} \phi\left(L^{2}(b-a)\left|\mathfrak{t}_{0}-u_{0}\right|\right)+\frac{\gamma_{K}}{\gamma_{0}} \phi\left(\frac{\left|K\left(\psi, v, u_{0}\right)\right|}{\gamma_{K}}\right)+\frac{\gamma_{J}}{\gamma_{0}} \phi\left(\frac{\left|J\left(\psi, v, v_{0}\right)\right|}{\gamma_{J}}\right),
\end{aligned}
$$

and so 


$$
\begin{aligned}
& \int_{a}^{b} \phi\left(\frac{\left|K\left(\psi, v, \mathfrak{u}_{1}(v)\right)\right|+\left|J\left(\psi, v, \mathfrak{u}_{2}(v)\right)\right|}{\gamma_{0}}\right) \mathrm{d} v \\
& \quad \leq \frac{L^{2}(b-a)^{2} \gamma_{1}}{\gamma_{0}} C_{1}+\frac{(b-a)}{\gamma_{0}} \phi\left(L^{2}(b-a)\left|\mathfrak{u}_{0}-u_{0}\right|\right)+\frac{\gamma_{K}}{\gamma_{0}} C_{2}+\frac{\gamma_{J}}{\gamma_{0}} C_{3} \equiv C_{0}<\infty .
\end{aligned}
$$

Now, it follows from (67) that

$$
\begin{aligned}
& \phi\left(\frac{1}{(b-a)} \int_{a}^{b} \frac{\left|K\left(\psi, v, \mathfrak{t}_{1}(v)\right)\right|+\left|J\left(\psi, v, \mathfrak{u}_{2}(v)\right)\right|}{\gamma_{0}} \mathrm{~d} v\right) \\
& \quad \leq \frac{1}{(b-a)} \int_{a}^{b} \phi\left(\frac{\left|K\left(\psi, v, \mathfrak{t}_{1}(v)\right)\right|+\left|J\left(\psi, v, \mathfrak{t}_{2}(v)\right)\right|}{\gamma_{0}}\right) \mathrm{d} v \leq \frac{C_{0}}{b-a},
\end{aligned}
$$

which implies

Similarly, if

$$
\int_{a}^{b}\left|K\left(\psi, v, \mathfrak{u}_{1}(v)\right)\right|+\left|J\left(\psi, v, \mathfrak{u}_{2}(v)\right)\right| \mathrm{d} \tau \leq \gamma_{0}(b-a) \phi^{-1} \frac{C_{0}}{b-a}<\infty .
$$

$$
\max \left\{\int_{a}^{b} \mathfrak{u t}_{1}^{\prime}(\psi) \mathrm{d} \psi+\left|\mathfrak{u}_{0}-u_{0}\right|, \int_{a}^{b} \mathfrak{u}_{2}^{\prime}(\psi) \mathrm{d} \psi+\left|\mathfrak{u}_{0}-v_{0}\right|\right\}=\int_{a}^{b} \mathfrak{u}_{2}^{\prime}(\psi) \mathrm{d} \psi+\left|\mathfrak{u}_{0}-v_{0}\right|,
$$

then

$$
\begin{aligned}
& \int_{a}^{b} \phi\left(\frac{\left|K\left(\psi, v, \mathfrak{u}_{1}(v)\right)\right|+\left|J\left(\psi, v, \mathfrak{u}_{2}(v)\right)\right|}{\gamma_{0}^{\prime}}\right) \mathrm{d} v \\
& \quad \leq \frac{L^{2}(b-a)^{2} \gamma_{2}}{\gamma_{0}^{\prime}} C_{1}^{\prime}+\frac{1}{\gamma_{0}^{\prime}} \phi\left(L^{2}(b-a)\left|\mathfrak{u}_{0}-v_{0}\right|\right)+\frac{\gamma_{K}}{\gamma_{0}^{\prime}} C_{2}+\frac{\gamma_{J}}{\gamma_{0}^{\prime}} C_{3} \equiv \frac{C_{0}^{\prime}}{b-a},
\end{aligned}
$$

implying

$$
\int_{a}^{b}\left|K\left(\psi, v, \mathfrak{u t}_{1}(v)\right)\right|+\left|J\left(\psi, v, \mathfrak{u}_{2}(v)\right)\right| \mathrm{d} v \leq \gamma_{0}^{\prime}(b-a) \phi^{-1}\left(\frac{C_{0}^{\prime}}{b-a}\right)<\infty
$$

Hence, in both the cases,

$$
\int_{a}^{b}\left|K\left(\psi, v, \mathfrak{u}_{1}(v)\right)\right| \mathrm{d} v+\int_{a}^{b}\left|J\left(\psi, v, \mathfrak{u}_{21}(v)\right)\right| \mathrm{d} v<\infty .
$$

Therefore, $K, J \in L^{1}[a, b]$. Thus, the operator $T$ is well defined on $\mathfrak{M}_{w}^{* 1} \times \mathfrak{M}_{w}^{* 1}$ and by (61), $T\left(\mathfrak{u}_{1}, \mathfrak{t}_{2}\right) \in A C[a, b]$ for all $\mathfrak{t}_{1}, \mathfrak{t}_{2} \in \mathfrak{M}_{w}^{* 1}$ which implies that $\left(T\left(\mathfrak{u}_{1}, \mathfrak{t}_{2}\right)\right)^{\prime} \in$ $L^{1}[a, b]$ and

$$
\left.\left.\left(T\left(\mathfrak{u}_{1}, \mathfrak{u}_{2}\right)\right)^{\prime}(\psi)=K\left(\psi, v, \mathfrak{t}_{1}(v)\right)\right)+J\left(\psi, v, \mathfrak{u}_{2}(v)\right)\right),
$$

for almost all $v \in[a, b]$.

Step 2. It is clear from (61) and $\mu(a)=\sigma(a)=a$ that given $\mathfrak{u}_{1}, \mathfrak{t}_{2}, \mathfrak{w} \in \mathfrak{M}_{w}^{* 1}$, 


$$
T\left(\mathfrak{u}_{1}, \mathfrak{u}_{2}\right)(a)=\int_{a}^{\mu(a)} K\left(\psi, v, \mathfrak{u}_{1}(v)\right) \mathrm{d} v+\int_{a}^{\sigma(a)} J\left(\psi, v, \mathfrak{t}_{2}(v)\right) \mathrm{d} v+\mathfrak{w}(a)=u_{0}
$$

and so $T\left(\mathfrak{u}_{1}, \mathfrak{u}_{2}\right) \in \mathfrak{M}^{1}=\left\{\mathfrak{u}:[a, b] \longrightarrow R: \mathfrak{u}(a)=u_{0}\right\}$. Now, we will show that $T\left(\mathfrak{u}_{1}, \mathfrak{u}_{2}\right) \in \mathfrak{M}_{w}^{*}$. By virtue of (59) and (73)-(82), we have

$$
w_{\gamma_{0}}\left(T\left(\mathfrak{t}_{1}, \mathfrak{u}_{2}\right), \mathfrak{u}_{0}\right)=\int_{a}^{b} \phi\left(\frac{\left|\left(T\left(\mathfrak{t}_{1}, \mathfrak{t}_{2}\right)\right)^{\prime}(v)\right|}{\gamma_{0}}\right) \mathrm{d} v \leq C_{0},
$$

or

$$
w_{\gamma_{0}^{\prime}}\left(T\left(\mathfrak{t}_{1}, \mathfrak{t}_{2}\right), \mathfrak{u}_{0}\right)=\int_{a}^{b} \phi\left(\frac{\left|\left(T\left(\mathfrak{u}_{1}, \mathfrak{u}_{2}\right)\right)^{\prime}(v)\right|}{\gamma_{0}}\right) \mathrm{d} v \leq C_{0}^{\prime},
$$

and so $T$ maps $\mathfrak{M}_{w}^{* 1} \times \mathfrak{M}_{w}^{* 1} \longrightarrow \mathfrak{M}_{w}^{* 1}$.

Step 3. To obtain (66), let $\gamma>0$ and $\mathfrak{t}_{1}, \mathfrak{t}_{2}, \mathfrak{t}_{3} \in \mathfrak{M}_{w}^{* 1}$. By (58), (59), and (82), we find

$$
\begin{aligned}
w_{L(b-a) \gamma}\left(T\left(\mathfrak{u}_{1}, \mathfrak{t}_{2}\right), T\left(\mathfrak{t}_{2}, \mathfrak{t}_{3}\right)\right) & =w_{L(b-a) \gamma}\left(T\left(\mathfrak{t}_{1}, \mathfrak{t}_{2}\right)-T\left(\mathfrak{t}_{2}, \mathfrak{t}_{3}\right), 0\right) \\
& =\int_{a}^{b} \phi\left(\frac{\left|\left(T\left(\mathfrak{t}_{1}, \mathfrak{t}_{2}\right)-T\left(\mathfrak{t}_{2}, \mathfrak{t}_{3}\right)\right)^{\prime}(v)\right|}{L(b-a) \gamma}\right) \mathrm{d} v \\
& =\int_{a}^{b} \phi\left(\frac{\left|\left[K\left(\psi, v, \mathfrak{t}_{1}(v)\right)+J\left(\psi, v, \mathfrak{t}_{2}(v)\right)\right]-\left[K\left(\psi, v, \mathfrak{t}_{2}(v)\right)+J\left(\psi, v, \mathfrak{t}_{3}(v)\right)\right]\right|}{L(b-a) \gamma}\right) \mathrm{d} v \\
& =\int_{a}^{b} \phi\left(\frac{\left|\left[K\left(\psi, v, \mathfrak{t}_{1}(v)\right)-J\left(\psi, v, \mathfrak{u}_{2}(v)\right)\right]+\left[K\left(\psi, v, \mathfrak{t}_{2}(v)\right)-J\left(\psi, v, \mathfrak{t}_{3}(v)\right)\right]\right|}{L(b-a) \gamma}\right) \mathrm{d} v .
\end{aligned}
$$

By (m2) and Lebesgue's theorem, we have for almost all $v \in[a, b]\left(\right.$ since $\left.\mathfrak{t}_{1}(a)=\mathfrak{t}_{2}(a)=\mathfrak{t}_{3}(a)=u_{0}\right)$,

$$
\left|\left[K\left(\psi, v, \mathfrak{t}_{1}(v)\right)+J\left(\psi, v, \mathfrak{t}_{2}(v)\right)\right]-\left[K\left(\psi, v, \mathfrak{t}_{2}(v)\right)+J\left(\psi, v, \mathfrak{t}_{3}(v)\right)\right]\right| \leq L^{2}(b-a) \max \left\{\left|\mathfrak{t}_{1}-\mathfrak{u}_{2}\right|,\left|\mathfrak{u}_{2}-\mathfrak{u}_{3}\right|\right\}
$$

Therefore,

$$
\begin{aligned}
& \left|\left[K\left(\psi, v, \mathfrak{t}_{1}(v)\right)+J\left(\psi, v, \mathfrak{u}_{2}(v)\right)\right]-\left[K\left(\psi, v, \mathfrak{u}_{2}(v)\right)+J\left(\psi, v, \mathfrak{t}_{3}(v)\right)\right]\right| \\
& \quad \leq L^{2}(b-a) \max \left\{\int_{a}^{b}\left(\mathfrak{t}_{1}-\mathfrak{t}_{2}\right)^{\prime}(\psi), \int_{a}^{b}\left(\mathfrak{t}_{2}-\mathfrak{u}_{3}\right)^{\prime}(\psi)\right\} .
\end{aligned}
$$


Applying (67) and (68), we get

$$
\begin{aligned}
& \phi\left(\frac{\left|\left[K\left(\psi, v, \mathfrak{t}_{1}(v)\right)-J\left(\psi, v, \mathfrak{t}_{2}(v)\right)\right]+\left[K\left(\psi, v, \mathfrak{t}_{2}(v)\right)-J\left(\psi, v, \mathfrak{t}_{3}(v)\right)\right]\right|}{L(b-a) \gamma}\right) \\
& \quad \leq \phi\left(L^{2}(b-a) \max \left\{\int_{a}^{b} \frac{\left(\mathfrak{t}_{1}-\mathfrak{t}_{2}\right)^{\prime}(\psi)}{L(b-a) \gamma} \mathrm{d} \psi, \int_{a}^{b} \frac{\left(\mathfrak{u}_{2}-\mathfrak{t}_{3}\right)^{\prime}(\psi)}{L(b-a) \gamma} \mathrm{d} v\right\}\right) \\
& \quad=\phi\left(L(b-a) \max \left\{\int_{a}^{b} \frac{\left(\mathfrak{u}_{1}-\mathfrak{t}_{2}\right)^{\prime}(\psi)}{L(b-a) \gamma} \mathrm{d} \psi, \int_{a}^{b} \frac{\left(\mathfrak{t}_{2}-\mathfrak{t}_{3}\right)^{\prime}(\psi)}{(b-a) \gamma} d v\right\}\right) \\
& \quad \leq L(b-a) \phi\left(\max \left\{\frac{1}{b-a} \int_{a}^{b} \frac{\left(\mathfrak{t}_{1}-\mathfrak{t}_{2}\right)^{\prime}(\psi)}{\gamma} \mathrm{d} \psi, \frac{1}{b-a} \int_{a}^{b} \frac{\left(\mathfrak{t}_{2}-\mathfrak{t}_{3}\right)^{\prime}(\psi)}{\gamma} \mathrm{d} v\right\}\right) \\
& \quad \leq L \max \left(\left\{\int_{a}^{b} \phi \frac{\left(\mathfrak{t}_{1}-\mathfrak{t}_{2}\right)^{\prime}(\psi)}{\gamma} \mathrm{d} \psi, \int_{a}^{b} \phi \frac{\left(\mathfrak{t}_{2}-\mathfrak{u}_{3}\right)^{\prime}(\psi)}{\gamma} \mathrm{d} \psi\right\}\right) \\
& \quad=L \max \left\{w_{\gamma}\left(\mathfrak{u}_{1}, \mathfrak{u}_{2}\right), w_{\gamma}\left(\mathfrak{t}_{2}, \mathfrak{u}_{3}\right)\right\} .
\end{aligned}
$$

Therefore,

$$
\begin{aligned}
& w_{L(b-a) \gamma}\left(T\left(\mathfrak{t}_{1}, \mathfrak{t}_{2}\right), T\left(\mathfrak{t}_{2}, \mathfrak{t}_{3}\right)\right) \\
& \quad=\int_{a}^{b} \phi\left(\frac{\left|\left[K\left(\psi, v, \mathfrak{t}_{1}(v)\right)-J\left(\psi, v, \mathfrak{t}_{2}(v)\right)\right]+\left[K\left(\psi, v, \mathfrak{t}_{2}(v)\right)-J\left(\psi, v, \mathfrak{t}_{3}(v)\right)\right]\right|}{L(b-a) \gamma}\right) \mathrm{d} v \\
& \quad \leq L(b-a) \max \left\{w_{\gamma}\left(\mathfrak{t}_{1}, \mathfrak{u}_{2}\right), w_{\gamma}\left(\mathfrak{t}_{2}, \mathfrak{u}_{3}\right)\right\} .
\end{aligned}
$$

Theorem 9. Under the assumptions ( $m 1),(m 2)$, and $0<L(b-a)<1$, integral equation (60) admits a solution $\mathfrak{u} \in G V_{\phi}[a, b]$.

Proof. (i) By Lemmas 1 and 2, $w$ is strict modular on

$$
\mathfrak{M}^{1}=\left\{\mathfrak{t} ; \mathfrak{t}:[a, b] \longrightarrow \mathfrak{A}, \mathfrak{t}(a)=\mathfrak{t}_{0}\right\},
$$

and the modular space

$$
\mathfrak{M}_{w}^{* 1}=G V_{\phi}([a, b]) \cap \mathfrak{M}^{1}=\left\{\mathfrak{u} \in G V_{\phi}([a, b]), \mathfrak{u}(a)=\mathfrak{u}_{0}\right\},
$$

is $w$-complete. By Theorem 8 , the operator $T: \mathfrak{M}_{w}^{* 1} \times \mathfrak{M}_{w}^{* 1} \longrightarrow \mathfrak{M}_{w}^{* 1}$ is Ciric-Presic type strongly $w$-contractive. We are left to show that for some $\gamma>0$, $\exists \mathfrak{u} \in \mathfrak{M}_{w}^{* 1}$, such that $w_{\gamma}(\mathfrak{t}, T \mathfrak{i})<\infty$. Clearly, for constant function $\mathfrak{t}_{0} \in \mathfrak{M}_{w}^{* 1}$, we have

$$
\begin{aligned}
w_{\gamma_{0}}\left(T\left(\mathfrak{u}_{0}, \mathfrak{t}_{0}\right), \mathfrak{u}_{0}\right) & =w_{\gamma}\left(T\left(\mathfrak{u}_{0}, \mathfrak{t}_{0}\right)-\mathfrak{u}_{0}, 0\right) \\
& =\int_{a}^{b} \phi\left(\frac{\left|T\left(\mathfrak{t}_{0}, \mathfrak{t}_{0}\right)-\mathfrak{t}_{0}\right|^{\prime}}{\gamma_{0}}\right) \mathrm{d} \tau \\
& =\int_{a}^{b} \phi\left(\frac{\left|T\left(\mathfrak{t}_{0}, \mathfrak{t}_{0}\right)\right|^{\prime}}{\gamma_{0}}\right) \mathrm{d} \tau \\
& =\int_{a}^{b} \phi\left(\frac{\left|K\left(\psi, v, \mathfrak{t}_{0}\right)\right|+\left|J\left(\psi, v, \mathfrak{t}_{0}\right)\right|}{\gamma_{0}}\right) \mathrm{d} \tau \\
& \leq \frac{L^{2}(b-a)^{2} \gamma_{1}}{\gamma_{0}} \int_{a}^{b} \phi\left(\frac{\left|\mathfrak{t}_{0}^{\prime}(\psi)\right|}{\gamma_{1}}\right) \mathrm{d} \psi+\frac{(b-a)}{\gamma_{0}} \phi\left(L^{2}(b-a)\left|\mathfrak{t}_{0}-u_{0}\right|\right)+\frac{\gamma_{K}}{\gamma_{0}} C_{2}+\frac{\gamma_{J}}{\gamma_{0}} C_{3} .
\end{aligned}
$$


Since $\mathfrak{u}_{0} \in \mathfrak{M}_{w}^{* 1}$ is a constant function, $\left(\mathfrak{t}_{0}\right)^{\prime}=0$. Therefore,

$$
\begin{aligned}
w_{\gamma_{0}}\left(T\left(\mathfrak{u}_{0}, \mathfrak{t}_{0}\right), \mathfrak{u}_{0}\right) \leq & \frac{b-a}{\gamma_{0}} \phi\left(L^{2}(b-a)\left|\mathfrak{t}_{0}-u_{0}\right|\right) \\
& +\frac{\gamma_{K}}{\gamma_{0}} C_{2}+\frac{\gamma_{J}}{\gamma_{0}} C_{3}<\infty,
\end{aligned}
$$

where $L^{2}(b-a) \gamma_{1}+1+\gamma_{K}+\gamma_{J}=\gamma_{0}$. By Theorem 4 , the integral operator $T$ admits a fixed point and thus problem (60) has a solution.

Theorem 10. Under the assumptions ( $m 1),(m 2)$, and $0<L(b-a)<1$, integral equation (60) is Ulam-Hyers stable.

Proof. Let $\epsilon>0$ and $u^{*} \in \mathfrak{M}_{w}^{* 1}$ for which $\int_{a}^{b} \phi\left(\mid u^{* \prime}\right.$ $(\tau)-K\left(\tau, v, u^{*}(\mu(\tau))-K\left(\tau, v, u^{*}(\sigma(\tau))-\mathfrak{w}^{\prime}(\tau) \mid / \gamma\right) \mathrm{d} \tau \leq \epsilon\right.$. Then, we have

$$
\begin{gathered}
\int_{a}^{b} \phi\left(\frac{\left|u^{*}(\tau)-T\left(u^{*}(\mu(\tau)), u^{*}(\sigma(\tau))\right)\right|^{\prime}}{\gamma}\right) \mathrm{d} \tau \leq \epsilon \\
\Longrightarrow w_{\lambda}\left(u^{*}-T\left(u^{*}, u^{*}\right), 0\right) \leq \epsilon \\
\Longrightarrow w_{\lambda}\left(u^{*}, T\left(u^{*}, u^{*}\right)\right) \leq \epsilon .
\end{gathered}
$$

By Theorem 8 , the operator $T: \mathfrak{M}_{w}^{* 1} \times \mathfrak{M}_{w}^{* 1} \longrightarrow \mathfrak{M}_{w}^{* 1}$ is Ciric-Presic type strongly $w$-contractive and so by Proposition $1, T$ is $c$-WPPO. By Theorem 7, the fixed-point equation (62) is Ulam-Hyers stable; that is, there exists $u \in \mathfrak{M}_{w}^{* 1}$ such that $u=T(u, u)$ and $\omega_{\lambda}\left(u^{*}, u\right) \leq c \epsilon$. Thus, we have

$$
\begin{aligned}
& \omega_{\lambda}\left(u^{*}, u\right) \leq c \epsilon \\
& \Longrightarrow \omega_{\lambda}\left(u^{*}-u, 0\right) \leq c \epsilon \\
& \Longrightarrow \int_{a}^{b} \phi\left(\frac{\left|u^{* \prime}(\tau)-u^{\prime}(\tau)\right|}{\gamma}\right) \mathrm{d} \tau \leq c \epsilon .
\end{aligned}
$$

We now furnish a numerical example to validate the hypothesis of Theorem 9.

Example 3. Consider the integral equation

$$
\begin{aligned}
x(s)= & -\frac{s}{8}+\frac{3 s^{2}}{4}+\frac{1}{4} \arctan \left(\frac{s}{2}\right)-\frac{1}{2} \arctan \left(\frac{s^{2}}{2}\right)-\frac{s^{2}}{8} \ln \left(1+\frac{s^{4}}{4}\right) \\
& +\int_{0}^{s^{2} / 2} \frac{\ln (1+x(v))}{4} \mathrm{~d} v+\int_{0}^{s / 2} \frac{x(v)}{4(1+x(v))} \mathrm{d} v, \quad s \in[0,1],
\end{aligned}
$$

for some $x \in \mathfrak{M}_{w}^{* 1}, \mathfrak{A}=[0,100]$, and $u_{0}=0$. We will apply $\quad$ Proof. $\quad$ Define the operator $T: \mathfrak{M}_{w}^{* 1} \longrightarrow \mathfrak{M}_{w}^{* 1}$ as Theorem 9, to prove the existence of a solution of (97).

$$
\begin{aligned}
\operatorname{Tx}(s)= & -\frac{s}{8}+\frac{3 s^{2}}{4}+\frac{1}{4} \arctan \left(\frac{s}{2}\right)-\frac{1}{2} \arctan \left(\frac{s^{2}}{2}\right)-\frac{s^{2}}{8} \ln \left(1+\frac{s^{4}}{4}\right) \\
& +\int_{0}^{s^{2} / 2} \frac{\ln (1+x(v))}{2} \mathrm{~d} v+\int_{0}^{s / 2} \frac{x(v)}{2(1+x(v))} \mathrm{d} v, \quad s \in[0,1] .
\end{aligned}
$$

Now, set $K(s, v, x(v))=(\ln (1+x(v)) / 4), J(s, v, x(v))=$ $(x(v) / 4(1+x(v))), \mu(s)=\left(s^{2} / 2\right), \sigma(s)=(s / 2)$, and $\mathfrak{w}(s)=$ $-(s / 8)+\left(3 s^{2} \quad / 4\right)+(1 / 4) \arctan (s / 2)-(1 / 2) \arctan \left(s^{2} / 2\right)-$ $\left(s^{2} / 8\right) \ln \left(1+\left(s^{4} / 4\right)\right)$. We observe the following: 


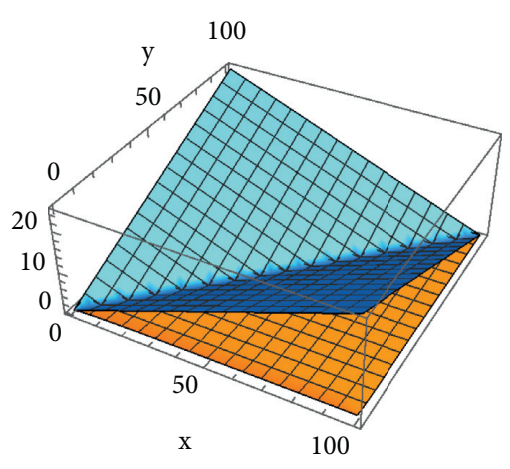

$|(1 / 4) \log (1+\mathrm{x})-(1 / 4) \log (1+\mathrm{y})|$ $|x-y| / 4$

FIGURE 1: Inequality in (99).

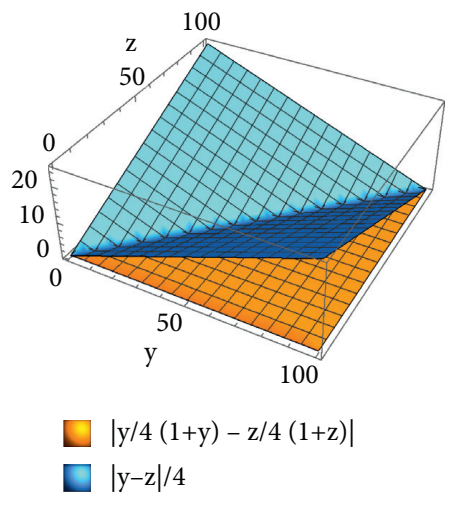

Figure 2: Inequality in (100).

(i) Let $\phi(t)=t$ for all $t \in R^{+}$. For $u_{0}=v_{0}=9$ and $\gamma_{K}=1, \gamma_{J}=1$, we see that the functions $K$ and $J$ satisfy (m1).

(ii) By using the Mathematica software, we found that the following inequalities are true for all $x, y, z \in[0,100]$ (see Figures 1 and 2):

$$
\begin{gathered}
\left|\frac{\ln (1+x)}{4}-\frac{\ln (1+y)}{4}\right| \leq \frac{|x-y|}{4}, \\
\left|\frac{y}{4(1+y)}-\frac{z}{4(1+z)}\right| \leq \frac{|y-z|}{4} .
\end{gathered}
$$

Thus, we get

$$
\begin{aligned}
& \mid K(s, v, x(v)-K(s, v, y(v)|+| J(s, v, y(v)-J(s, v, z(v) \mid \\
& \quad \leq\left|\frac{\ln (1+x)-\ln (1+y)}{4}\right|+\left|\frac{y}{4(1+y)}-\frac{z}{4(1+z)}\right| \\
& \quad \leq \frac{1}{2} \max \{|x-y|,|y-z|\},
\end{aligned}
$$

and thus, $K$ and $J$ satisfies $(\mathrm{m} 2)$, with $L^{2}=(1 / \sqrt{2})$.
Hence, all the conditions of Theorem 9 are satisfied. It is evident that integral equation (97) has a unique solution $x \in \mathfrak{M}_{w}^{* 1}$ defined by $x(s)=s^{2}$.

\section{Conclusion}

The fixed-point technique is used to solve many mathematical problems as it gets involved with differential and integral equations, integro-differential equations, game theory, economics, and more disciplines. In this work, unique fixed points of generalised Presic type operators defined on modular metric space are obtained and the results are applied in proving the existence of solutions of integral equations involving Caratheodory type functions. Ulam-Hyers stability of fixed-point equations involving Presic type operators in a modular metric space is introduced. Our results pave way for further research in modular metric spaces. Following the techniques of our work, Presic-Bogin-type contractions, Presic-Chatterjee type contractions, and Presic-Hardy-Rogers type contractions can be extended to the setting of a modular metric space.

\section{Data Availability}

No data were used to support this study.

\section{Conflicts of Interest}

The authors declare that they have no conflicts of interest.

\section{Acknowledgments}

This research was supported by the Deanship of Scientific Research, Prince Sattam bin Abdulaziz University, Alkharj, Saudi Arabia, under Grant no. 2020/01/17320.

\section{References}

[1] M. Fréchet, "Sur quelques points du calcul fonctionnel," Rendiconti del Circolo Matematico di Palermo, vol. 22, p. 172, 1906.

[2] F. Hausdorff, "Grundzuge der Mengenlehre," Monatshefte fr Mathematik und Physik, vol. 26, Article ID A34A35, 1914.

[3] I. A. Bakhtin, "The contraction mapping principle in quasimetric spaces," Funct. Anal. Unianowsk Gos. Ped. Inst, vol. 30, pp. 26-37, 1989.

[4] A. Branciari, "A fixed point theorem of Banach-Caccioppoli type on a class of generalized metric spaces," Publicationes Mathematicae Debrecen, vol. 57, pp. 31-37, 2000.

[5] R. George, S. Radenovic, K. P. Reshma, and S. Shukla, "Rectangular $b$-metric spaces and contraction principle," The Journal of Nonlinear Science and Applications, vol. 8, no. 6, pp. 1005-1013, 2015.

[6] Z. D. Mitrović and S. Radenović, "The Banach and Reich contractions in $b_{v}(s)$-metric spaces," Journal of Fixed Point Theory and Applications, vol. 19, 2017.

[7] W. M. Kozlowski, Modular Function Spaces, Dekker, New York, NY, USA, 1988.

[8] M. A. Khamsi, W. M. Kozlowski, and S. Reich, "Fixed point theory in modular function spaces," Nonlinear Analysis: Theory, Methods \& Applications, vol. 14, no. 11, pp. 935-953, 1990. 
[9] V. V. Chistyakov, "Modular metric spaces, I: basic concepts," Nonlinear Analysis, vol. 72, p. 114, 2010.

[10] V. V. Chistyakov, "A fixed point theorem for contractions in modular metric spaces," 2011, https://arxiv.org/abs/1112. 5561.

[11] A. Umit, E. Karapinar, and M. E. Inci, "Fixed point theorems in complete modular metric spaces and an application to antiperiodic Boundary value problems," Filomat, vol. 31, no. 17, pp. 5475-5488, 2017.

[12] P. Chaipunya, Y. J. Cho, and P. Kumam, "Geraghty-type theorems in modular metric spaces with an application to partial differential equation," Advances in Difference Equations, vol. 83, 2012.

[13] D. Turkoglu and E. Kilinc, "Some fixed point results for Caristi type mappings in modular metric space with application," International Journal of Analysis and Applications, vol. 12, no. 1, pp. 15-21, 2016.

[14] M. Paknazar, M. Eshaghi, Y. J. Cho, and S. M. Vaezpour, “A Pata-type fixed point theorem in modular spaces with application," Fixed Point Theory and Applications, vol. 2013, no. 1, p. 239, 2013.

[15] P. Chaipunya, C. Mongkolkeha, W. Sintunavarat, and P. Kumam, "Fixed point theorems for multivalued mappings in modular metric spaces," Abstract and Applied Analysis, vol. 2012, Article ID 503504, 14 pages, 2012.

[16] P. Chaipunya, C. Mongkolkeha, W. Sintunavarat, and P. Kumam, "Erratum to Fixed point theorems for multivalued mappings in modular metric spaces," Abstract and Applied Analysis, vol. 2012, Article ID 241919, 2 pages, 2012.

[17] A. A. N. Abdou, "Some fixed point theorems in modular metric spaces," The Journal of Nonlinear Science and Applications, vol. 9, Article ID 43814387, 2016.

[18] E. Feizi and Z. Hosseini, "Fixed point theorems for new J-type mappings in modular spaces," International Journal of Nonlinear Analysis and Applications, vol. 10, no. 1, Article ID 111118, 2019.

[19] L. Fatemeh, S. Naeem, G. Liliana, and M. F. Bota, "Nadler's theorem in incomplete modular space," Mathematics, vol. 9, no. 1927, 2021.

[20] L. Fatemeh, S. Naeem, and M. Abbas, "On the fixed points of multivalued mappings in b-metric spaces and their application to linear systems," UPB Scientific Bulletin, Series A, vol. 82, no. 4, 2020.

[21] S. B. Presic, "Sur la convergence des suites," Comptes Rendus de l Académie des Sciences, vol. 260, pp. 3828-3830, 1965.

[22] S. B. Presic, "Sur une classe dinequations aux differences finite et sur la convergence de certaines suites," Publications de l'Institut Mathématique, vol. 5, no. 19, pp. 75-78, 1965.

[23] L. B. Ciric and S. B. Presic, "On Presic type generalisation of Banach contraction principle," Acta Mathematica Universitatis Comenianae, vol. LXXVI, no. 2, pp. 143-147, 2007.

[24] K. P. R. Rao, M. Ali, and B. Fisher, "Some presic type generalizations of the Banach contraction principle," Mathematica Moravica, vol. 15, no. 1, pp. 41-47, 2011.

[25] M. Pacurar, "Common fixed points for almost Presic type operators," Carpathian Journal of Mathematics, vol. 28, no. 1, pp. 117-126, 2012.

[26] M. S. Khan, M. Berzig, and B. Samet, "Some convergence results for iterative sequences of Prešić type and applications," Advances in Difference Equations, vol. 2012, no. 1, 2012.

[27] R. George, K. Reshma, and R. Rajagopalan, "A generalised fixed point theorem of Presic type in cone metric spaces and application to Markov process," Fixed Point Theory and Applications, vol. 2011, no. 1, 2011.
[28] S. Shukla and R. Sen, "Set-valued Prešić-Reich type mappings in metric spaces," Revista de la Real Academia de Ciencias Exactas, Fisicas y Naturales. Serie A. Matematicas, vol. 108, no. 2, pp. 431-440, 2012.

[29] H. K. Pathak, R. George, H. A. Nabwey, M. S. El-Paoumy, and K. P. Reshma, "Some generalized fixed point results in a b-metric space and application to matrix equations," Fixed Point Theory and Applications, vol. 2015, no. 1, 2015.

[30] I. A. Rus, "Remarks on Ulam stability of the operational equations," Fixed Point Theory, vol. 10, no. 2, pp. 305-320, 2009.

[31] X. Li, A. Hussain, M. Adeel, and E. Savas, "Fixed point theorems for $\$\{\mid \text { mathscr }\{Z\}\}_{-}\{\mid$vartheta $\}$-contraction and applications to nonlinear integral equations $L_{v}$-contraction and applications to nonlinear integral equations," IEEE Access, vol. 7, pp. 120023-120029, 2019.

[32] E. A. Abdullah, A. Hussain, I. Muhammad, and A. Jamshaid, "Simulative suzuki-gerghaty type contraction with C -class functions and applications," IEEE Access, vol. 7, pp. 7528475291, 2019.

[33] B. Mouffak, B. Soufyane, and J. N. Juan, "Existence and Ulam stability for nonlinear implicit differential equations with Riemann-Liouville fractional derivative," Demonstratio Mathematica, vol. 52, Article ID 437450, 2019.

[34] V. V. Chistyakov, "Selections of bounded variation," Journal of Applied Analysis, vol. 10, no. 1, p. 182, Article ID MR2081486, 2004.

[35] V. V. Chistyakov, Metric Modular Spaces, Theory and Applications, Springer International Publishing, Berlin, Germany, 2015. 